\title{
The Finite Element Implementation of a K.P.P. Equation for the Simulation of Tsetse Control Measures in the Vicinity of a Game Reserve
}

\author{
S. J. Childs \\ ARC-Onderstepoort Veterinary Institute, Private Bag X5, \\ Pretoria, 0110, South Africa. \\ Tel: +27 72 8459556. E-mail: simonjohnchilds@gmail.com
}

Mathematical Biosciences, 227: 29-43, 2010

\begin{abstract}
An equation, strongly reminiscent of Fisher's equation, is used to model the response of tsetse populations to proposed control measures in the vicinity of a game reserve. The model assumes movement is by diffusion and that growth is logistic. This logistic growth is dependent on an historical population, in contrast to Fisher's equation which bases it on the present population. The model therefore takes into account the fact that new additions to the adult fly population are, in actual fact, the descendents of a population which existed one puparial duration ago, furthermore, that this puparial duration is temperature dependent. Artificially imposed mortality is modelled as a proportion at a constant rate. Fisher's equation is also solved as a formality.

The temporary imposition of a $2 \% \mathrm{day}^{-1}$ mortality everywhere outside the reserve for a period of 2 years will have no lasting effect on the influence of the reserve on either the Glossina austeni or the G. brevipalpis populations, although it certainly will eradicate tsetse from poor habitat, outside the reserve. A $5 \mathrm{~km}$-wide barrier with a minimum mortality of $4 \%$ day $^{-1}$, throughout, will succeed in isolating a worst-case, G. austeni population and its associated trypanosomiasis from the surrounding areas. A more optimistic estimate of its mobility suggests a mortality of $2 \%$ day $^{-1}$ will suffice. For a given target-related mortality, more mobile species are found to be more vulnerable to eradication than more sedentary species, while the opposite is true for containment.
\end{abstract}

Keywords: Kolmogoroff-Petrovsky-Piscounoff; K.P.P.; Fisher's equation; tsetse; Glossina brevipalpis; Glossina austeni; trypanosomiasis; congolense; vivax. 


\section{Introduction}

Various testse control measures, in the vicinity of a game reserve, are experimented with in a simulation context. The simulation models migration as diffusion, assumes growth is logistic and any artificially imposed mortality is taken to be a proportion at a constant rate. The problem posed is essentially one of designing counter measures against the influence of a reserve, of a particular size and geometry, on tsetse population levels outside its confines. The animals in the reserve are, moreover, considered to be a reservoir of more lethal strains of trypanosomiasis, as well as having a generally higher prevalence. The G. brevipalpis and G. austeni populations in and around the Hluhluwe-iMfolozi Game Reserve are the subject of this case study.

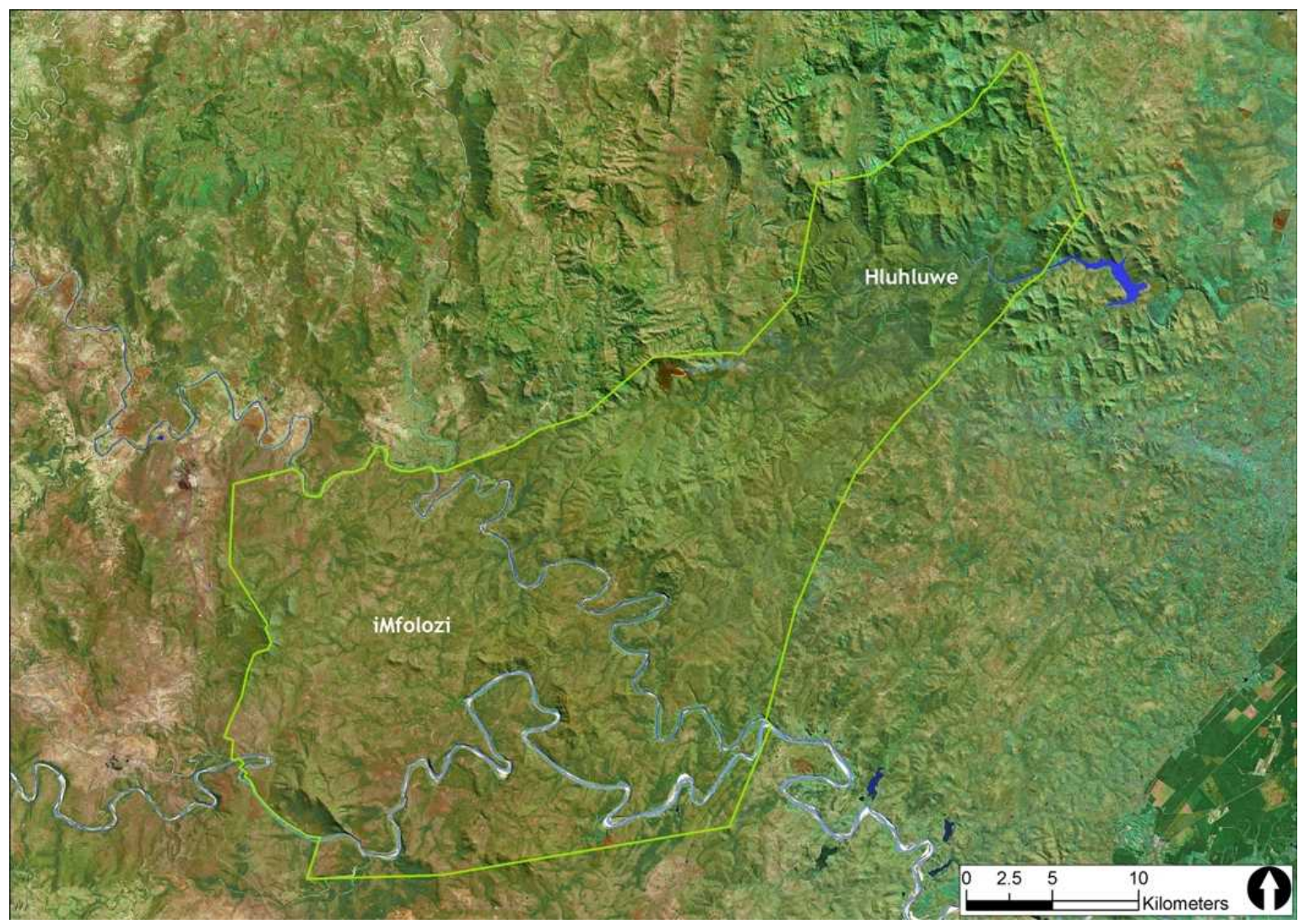

Figure 1: Satellite image of the Hluhluwe-iMfolozi Game Reserve and its surroundings.

The Hluhluwe-iMfolozi Game Reserve has the distinction of being the oldest proclaimed game reserve in Africa. It measures some $960 \mathrm{~km}^{2}$ and is located in the southern vicinity of $28{ }^{\circ} \mathrm{S}$ and $32^{\circ} \mathrm{E}$, in KwaZulu-Natal, South Africa. Inland of the coastal plain and set in the foothills of the escarpment, the temperature of the region is somewhat elevated for its latitutde. Riverine forest and thicket make the reserve the habitat of both G. brevipalpis and G. austeni. It is noteworthy, with regard to both species, that the Hluhluwe River has a flood plain within the reserve and that the backwater of the Hluhluwe Dam also extends well into it. At around this position, the reserve is approximately only $25 \mathrm{~km}$ from the St. Lucia estuary; lush habitat and a world 
heritage site which lies to the east. Habitat outside the reserve is otherwise degraded to the extent that the boundary of the reserve is discernable in satellite images. The presence of tsetse in association with large populations of buffalo and other wild animals, lead the HluhluweiMfolozi Game Reserve to be a thorn in the side of neighbouring agriculture. Any tsetse control within the reserve confines is, however, considered decidedly unwelcome nowadays, despite the reserve having its origins in an experimental area for the control of G. pallidipes ${ }^{1}$.

Much has recently come to light on the vector competence of $G$. brevipalpis and G. austeni. The predominant infection is that of Trypanasoma congolense, T. vivax being prevalent to a far lesser extent. It is noteworthy that out of $900 \mathrm{G}$. brevipalpis tenerals split into 3 equal groups and respectively allowed to feed on a different parasitaemic animal, the midgut of $4 \%$ and the preboscis of $0 \%$ were found to be infected (Motloang, Masumu, Van Den Bossche, MAJIWA and LATIF [19]). The prevalence of preboscal infection for the same experiment involving G. austeni tenerals was, in contrast, $12 \%$ and $19 \%$ were found to have an infected midgut (MOTLOANG ET AL. [19]). The same authors also conducted a second experiment in which they challenged each of 7 susceptable bovines with a different G. brevipalpis catch, taken from the wild, in an insect-proof facility (the combined catches totalling 468 specimens). No infection resulted. The same trial was then conducted by challenging each of 2 bovines and 1 goat with a different $G$. austeni catch taken from the wild (the combined catches totalling a mere 43 specimens). All three challenges resulted in infection. G. austeni is therefore a highly competent vector, while both prevalence and transmission rates are virtually non-existant in the case of G. brevipalpis. The issue of mechanical infection by G. brevipalpis is currently under investigation by the same authors.

Williams, Dransfield and BRIGHTWELl [29] originally entertained the idea of using Fisher's equation to model the distribution of tsetse populations and HARGROVE [11] devised the best implementation his circumstances permitted. The model entertained in this work is based on a very similar equation and differs mostly in the exact specification of population density in the logistic part. It belongs to a more general category of partial differential equations known as Kolmogoroff-Petrovsky-Piscounoff (K.P.P.) equations. Such partial differential equations also happen to be parabolic. In this regard, it is important to note that one cannot simply solve a parabolic, partial differential equation using an explicit method, nor should one apply the standard finite difference method to non-rectangular domains. The former is widely accepted as a faux pas and even in the event of circumstances which favour a correct solution, it has no credibility whatsoever. As such, the problem is ideally suited to the application of the finite element method in an implementation which is fully implicit in time. Fisher's equation is both parabolic and nonlinear.

In the present model flies are assumed to migrate by some kind of Brownian motion, down a diffusion gradient, based on the random nature of their movement (observed by BURSELL [2] and demonstrated by ROGERS [24]). Growth in the present fly population has its origins in an historical fly population; one which existed one puparial duration ago. This puparial duration is temperature dependent. Any artificially imposed mortality is modelled as a straight-

\footnotetext{
${ }^{1}$ The reserve falls within what was once the very extensive habitat of $G$. pallidipes, a species completely eradicated from KwaZulu-Natal in the first half of the 20th century.
} 
forward proportion at a constant rate. The model itself exceeded 5000 lines of fairly extensively commented Fortran, while the mesh generator exceeded 1700 lines.

Fisher's equation was also solved as a formality and from a point of view of academic interest. Fisher's equation is not perfectly suited to tsetse application owing to the large puparial duration which characterises the Glossina genus. A logistic term dependent on the present population density is something known to be incorrect when, in actual fact, the larval deposition, responsible for growth in the present population, took place a significant time previously. In the Fisher's-equation model the existance of the pupal phase is denied, alternatively, pupae are assumed to migrate and reproduce.

\section{Derivation of a Model}

The aim of the model is to predict how a tsetse population becomes distributed in space and how this distribution changes over time, through migration, self-regulating growth and artificially imposed mortality. The intention is to predict a population density, $\rho(\mathbf{x}, t)$ (in which $\mathbf{x}$ and $t$ are space and time respectively), based on these phenomena.

Which population? The subject of the intended model is the vector of trypanosomiasis, namely adult tsetse flies. Pupae neither migrate, nor do they (or any flies belonging to the pre-ovulatory cohort, for that matter) form any part of the actively reproductive population. For these reasons the population density, $\rho(\mathbf{x}, t)$, is defined not to include pupae. While it is tempting to also exclude any flies belonging to the pre-ovulatory cohorts from a reproductive point of view, such flies are mobile and subject to the external, artificial mortality to be imposed; indeed, the subject of this investigation. While the correspondence of the reproductive population to the mobile and vulnerable population is not perfect, it is suitably close.

With the relevant population identified, how might one model its change brought about through migration, self-regulating growth and artificially imposed mortality? If all three dynamics can be regarded as being mutually independent of one another, they can be considered in isolation.

\section{Migration}

BURSELL [2] put forward the theory that the movement of tsetse was of a random nature, not unlike Brownian motion, and ROGERS [24] proved these assertions quantitatively. If one can conceive of a gas as a continuum, it is only slightly more abstract to conceive of a fly population as a continuum. Consider the hypothetical scenario of a mobile population in the absence of either reproduction or mortality. Biomass should therefore be conserved and the standard continuum-mechanical result for mass conservation pertains. It can be manipulated to give a result not unlike the Reynolds transport theorem and Fick's first law applied. (A full exposition is provided in the addendum.) The resulting rate for the effect of migration, in 
isolation, is

$$
\frac{\partial \rho}{\partial t}=\lambda \operatorname{div} \nabla \rho
$$

in which $\lambda$ is the diffusion coefficient.

\section{Self-Regulating Growth}

The logistic model needs little introduction to a biological audience. The population is assumed to grow at a rate which is some proportion, $r$, of the parent population, $\rho^{*}$, and this growth rate must necessarily also be constrained by the carrying capacity, $K$, of the environment. The logistic rate for self-regulating growth, in isolation, is

$$
\frac{\partial \rho}{\partial t}=r \rho^{*}\left(1-\frac{\rho^{*}}{K}\right) .
$$

Which is the relevant population? Larval production is clearly dependent on the parental population which existed one puparial duration ago in the tsetse context. What about the population density in the second factor of the logistic term; the one limiting the growth rate? The pertinent population is not as obvious in this instance. Combined pupal and teneral mortality is an order of magnitude higher than adult mortality (HARGROVE [12]) and VALE [27] seems to think that parasitism alone accounts for between $40 \%$ to $60 \%$ of the overall pupal mortality, under usual circumstances and in a favourable environment. Quantitative work linking predation and parasitism to the density at pupal sites has been carried out by ROGERS and RANDOLPH [25]. That work could therefore be taken to recommend a logistic term based entirely on an historical population density, that which existed at the time of parturition.

Can such a model be reconciled with the other, remaining causes of pupal mortality? Although fat loss ${ }^{1}$ and water loss ${ }^{1}$ are determined by the external variables of temperature and humidity, an indirect dependence on population density is possible in the event of a shortage of available breeding sites. The spatial variation of temperature and humidity are otherwise incorporated in the carrying capacity and growth rate of each environment. It is important to note, however, that any temporal variation in the growth rate is beyond the scope of the standard logistic model, although such a model does allow for a time-dependent carrying capacity.

The fact that an historical population level was responsible for both larval production and subsequent, density-dependent mortality is taken into account in this particular model. Both larval production and subsequent natural mortality are assumed dependent on the historic population level, that which existed one puparial duration ago. The population density at the time of larval deposition was

$$
\rho^{*}(\mathbf{x}, t) \equiv \rho(\mathbf{x}, t-\bar{\tau})
$$

in which $\bar{\tau}$ is the relevant puparial duration.

\footnotetext{
${ }^{1}$ Teneral mortality from both fat loss and water loss is thought to be high and is often the cumulative effect of temperature and humidity conditions which prevailed during the pupal phase.
} 


\section{Artificially Imposed Mortality}

Suppose that the effect of targets, pour-ons etc. is to cause the population density to decline according to $\rho \delta t$, where $\delta$ is independent of the population density. Then the resulting rate for an artificially imposed mortality, in isolation, is

$$
\frac{\partial \rho}{\partial t}=-\delta \rho
$$

\subsection{A Governing Equation}

The combined effect of all three phenomena is additive and a model can therefore be based on the following equation. Two alternatives arise based on the exact specification of the parent population density, $\rho^{*}(\mathbf{x}, t)$. In the equation

$$
\frac{\partial \rho(\mathbf{x}, t)}{\partial t}=\lambda \operatorname{div} \nabla \rho(\mathbf{x}, t)+r \rho^{*}(\mathbf{x}, t)\left(1-\frac{\rho^{*}(\mathbf{x}, t)}{K}\right)-\delta \rho(\mathbf{x}, t),
$$

$\rho(\mathbf{x}, t)$ is otherwise the current population density (in which $\mathbf{x}$ and $t$ are space and time respectively), $\lambda$ is a diffusion rate, $r$ is the population growth rate, $K$ is the carrying capacity of the environment and $\delta$ is an artificially imposed mortality. The quantity $\rho^{*}(\mathbf{x}, t)$ is either an historical population density or the current population density, depending on the model preferred.

REMARK: Notice that in the special case of $\rho^{*}(\mathbf{x}, t)=\rho(\mathbf{x}, t)$ and $\delta=0$, Equation 1 becomes immediately recognizable as Fisher's equation in its classical form. It is otherwise part of a more general and widely inclusive family, known as Kolmogoroff-Petrovsky-Piscounoff equations.

\section{Limitation}

The correspondence of the reproductive population to the mobile and vulnerable population is not perfect. The modelled population includes pre-ovulatory flies which have a slightly longer interlarval period. These pre-ovulatory flies are not breeding, yet they are involved in logistic growth. An assumption implicit in the logistic model is therefore a fixed age profile.

\section{ASSUMPTION 1 The age profile of the population is fixed.}

How reasonable is this assumption? One consequence of any artificially imposed, adultselective mortality (such as is contemplated) is that a smaller proportion of reproductive adults should exist than the model supposes. This gives rise to a damped logistic response from the model at population levels above $K / 2$ and an over-reactive one for population levels below $K / 2$. 


\section{Implementation}

For the purposes of an implementation, Equation 1 can be rewritten in dimensionless form and the resulting primitive variable formulation converted to a variational one,

$$
\int_{\Omega} w \frac{\partial \rho}{\partial t} d \Omega+\frac{\lambda}{\lambda_{\text {scale }}} \int_{\Omega} \nabla w \cdot \nabla \rho d \Omega=\frac{r}{r_{\text {scale }}} \int_{\Omega} w \rho^{*}\left(1-\frac{\rho^{*}}{K}\right) d \Omega-\frac{\delta}{r_{\text {scale }}} \int_{\Omega} w \rho d \Omega
$$

(a full exposition is provided in the addendum). A fully implicit, backward difference is used for the temporal discretisation, while the finite element method is used for the spatial discretisation.

The solution at time $t$ is accordingly assumed to be a linear combination of shape functions, $\psi(\mathbf{x})$. That is,

$$
\left.\rho(\mathbf{x})\right|_{t}=\sum_{i=1}^{N} c_{i} \psi_{i}(\mathbf{x})
$$

where the $c_{i}$ are the constants of the finite element approximation (the nodal solutions) and $N$ is the total number of nodes. The problem on each element is calculated in terms of a standard, master element coordinate system, $\{\xi\}$. The approximate equation, to be solved for the nodal population densities, $P_{j}^{e}$ (pertaining to element $e$ ), is consequently

$$
\begin{gathered}
\mathbf{A}_{e=1}^{E}\left\{\frac{1}{\Delta t} \int_{\hat{\Omega}} \phi_{i} \phi_{j} J^{e} d \hat{\Omega}+\frac{\lambda}{\lambda_{\text {scale }}} \int_{\hat{\Omega}} \frac{\partial \phi_{i}}{\partial x_{k}} \frac{\partial \phi_{j}}{\partial x_{k}} J^{e} d \hat{\Omega}+\frac{\delta}{r_{\text {scale }}} \int_{\hat{\Omega}} \phi_{i} \phi_{j} J^{e} d \hat{\Omega}\right\} \mathbf{A}_{e=1}^{E} P_{j}^{e} \\
=\mathbf{A}_{e=1}^{E}\left\{\left.\frac{1}{\Delta t} \int_{\hat{\Omega}} \phi_{i} \phi_{m} J^{e} d \hat{\Omega} P_{m}^{e}\right|_{t-\Delta t}+\left.\frac{r}{r_{\text {scale }}} \int_{\hat{\Omega}} \phi_{i} \phi_{n} J^{e} d \hat{\Omega} P_{n}^{e}\right|_{t-\bar{\tau}}\right. \\
\left.-\left.\left.\frac{r}{r_{\text {scale }}} \int_{\hat{\Omega}} \phi_{i} \phi_{l} \frac{\phi_{j}}{K} J^{e} d \hat{\Omega} P_{l}^{e}\right|_{t-\bar{\tau}} P_{j}^{e}\right|_{t-\bar{\tau}}\right\},
\end{gathered}
$$

in which $A$ is the element assembly operator, $E$ is the total number of elements, $e$, into which the domain has been subdivided, $\hat{\Omega}$ is the master element domain, $\Delta t$ is the length of the time step, the $\phi_{i}(\xi)$ are the basis functions,

$$
\frac{\partial \phi_{i}}{\partial x_{j}}(\xi)=\frac{\partial \phi_{i}}{\partial \xi_{k}} \frac{\xi_{k}}{\partial x_{j}}, \quad J^{e}=\left|\frac{\partial \mathbf{x}}{\partial \boldsymbol{\xi}}\right| \text { for element } \mathrm{e},
$$

$\lambda$ is the rate of diffusion, $\lambda_{\text {scale }}$ is a diffusion rate scale, $r$ is the population growth rate, $r_{\text {scale }}$ is a population growth rate scale, $K$ is the carrying capacity of the environment and $\delta$ is an artificially imposed mortality. $\left.P_{n}^{e}\right|_{t-\bar{\tau}}$ denotes the solution at the time of larval deposition (that which led to the present eclosion), $\bar{\tau}$ being the relevant average of puparial durations. The second order accurate linearisation originally used in CHILDS [3],

$$
\left.2 \mathbf{P}^{e}\right|_{t-\Delta t}-\left.\mathbf{P}^{e}\right|_{t-2 \Delta t},
$$

was used for the first iteration of the nonlinear term arising in the analogous implementation of Fisher's equation. 


\section{The Relevant Parental Population}

The relevant parental population is that which existed one puparial duration ago. Determining the puparial duration leading to the present eclosion is a minor problem in its own right. It is known that at a given temperature, $T$, the puparial duration, in days, can be calculated according to the formula

$$
\tau(T)=\frac{1+e^{a+b T}}{\kappa},
$$

(Phelps AND Burrows [22]). For females, $\kappa=0.057 \pm 0.001, a=5.5 \pm 0.2$ and $b=$ $-0.25 \pm 0.01$. For males, $\kappa=0.053 \pm 0.001, a=5.3 \pm 0.2$ and $b=-0.24 \pm 0.01$ (HARGROVE [12]). The puparial durations of all species, with the exception of G. brevipalpis, are thought to lie within $10 \%$ of the value predicted by this formula (PARKER [21]). G. brevipalpis takes a little longer. The shortest puparial duration is that of G. austeni.

If $\bar{\tau}$ is the relevant average of puparial durations (which is, of course, dependent on itself) then

$$
\begin{aligned}
\bar{\tau} \equiv \frac{1}{\bar{\tau}}\left[[t-\text { floor }\{t\}] \tau\left(T_{\text {ceil }\{t\}}\right)\right. & +\sum_{i=\text { floor }\{t\}}^{\text {ceil }\{t-\bar{\tau}+1\}} \tau\left(T_{i}\right) \\
& \left.+[\operatorname{ceil}\{t-\bar{\tau}\}-(t-\bar{\tau})] \tau\left(T_{\text {ceil }\{t-\bar{\tau}\}}\right)\right],
\end{aligned}
$$

in which $\tau(T)$ is given by the formula Equation 2 and $T$ is the mean daily temperature on the day indicated by the subscript. Newton's method is used in solving the above equation. The relevant parental population at the time $t-\bar{\tau}$ is a weighted average of the nearest two solutions since a backward difference was used for the temporal discretisation.

\section{Application of the Model to Hluhluwe-iMfolozi}

G. brevipalpis and G. austeni are, in all likelihood, not the most suitable species for the application of such a model. This is since both forest species are thought to be fairly specialised and habitat-specific. This observation is independently born out by the ROGERS and ROBINSON [23] study (based on FORD and KATONDO [9]'s maps) as well as the pupal water loss model in CHILDS [4]. G. brevipalpis would, more generally, appear to be regionally associated with the riverine forest, or thicket, adjacent to drainage lines. While its pupal habitat appears to be more stringently confined than that of G. austeni (CHILDS [4]), the present work will suggest $G$. brevipalpis to be more far-ranging. G. austeni would, in contrast, appear to be relatively sedentary and less restricted by habitat. Nothing appears to be known about the diffusion rates of either G. brevipalpis or G. austeni and so-called worst-case values must be assumed. RoGERS' [24] experiments with $G$. fuscipes fuscipes were in fairly uniform habitat and even then there was light-sensitive preference. Little of relevance is otherwise known of G. brevipalpis and G. austeni. Their puparial durations subscribe the worst to the formula Equation 2 (PARKER 
[21]). G. austeni's puparial duration is the shortest and G. brevipalpis' is, by far, the longest. One would certainly prefer to be modelling savannah species. A strong affinity to habitat does, nonetheless, present a certain opportunity in ellucidating diffusion rates, as will presently be demonstrated.

Pertinent carrying capacities, growth rates and diffusion coefficients need to be associated with the nodes of the finite element mesh. The initial Hluhluwe-iMfolozi case study does not attempt to mimic reality to exactness. Instead, it is rather simplistic and more humble than the competancy of the model itself allows.

\subsection{The Carrying Capacity, $K$}

The Glossina genus is a $K$-strategist and carrying capacities are therefore important. Figure 2 is primarily concerned with distribution. The suggestion is, nevertheless, that a tsetse haven with a zone of influence is a premise on which to proceed. The G. brevipalpis data are certainly indicative of such a reality. The probability of $G$. austeni occurring within the reserve reaches a maximum of around 0.75 and one might speculate a smaller range due to the small size of this fly. The influence of minor habitat existing to the east of the reserve might therefore be disregarded on such a basis.

\begin{tabular}{|c|c|c|c|c|c|}
\hline & $\approx d / \mathrm{km}$ & $d \leq 0$ & $0<d \leq 2.5$ & $2.5<d \leq 5$ & $d>5$ \\
\hline \multirow{2}{*}{$K / \%$} & G. brevipalpis & 100 & 20 & 10 & 5 \\
\hline & G. austeni & 75 & 20 & 10 & 5 \\
\hline
\end{tabular}

Table 1: Modelled G. brevipalpis and G. austeni carrying capacities designated according to the approximate distance, $d$, from the reserve boundary.

A lack of data and the simplicity of an initial case study were deemed to vindicate such a simplistic approach. Note that it includes the assumption that $K$ is constant in time.

\subsection{The Maximum Growth Rate, $r$}

At Hluhluwe-iMfolozi mean annual temperatures, each female would produce four pupae based on GLASGOW [10]'s 49-day, average, adult life-span. The population would therefore grow by $2.8 \% \%^{-1}$ day $^{-1}$ in the absence of any early mortality, assuming an equal ratio of the sexes. Of course, in the real world there is massive pupal and teneral mortality. In reality, the 
growth rate is probably closer to $0.85 \%$ day $^{-1}$ (HARGROVE [12]). A logistic growth rate of $1.7 \%$ day $^{-1}$ was used for good habitat. One of the limitations of a logistic model is that $r$ is constant in time.

\begin{tabular}{l|cccc}
$\approx d / \mathrm{km}$ & $d \leq 0$ & $0<d \leq 2.5$ & $2.5<d \leq 5$ & $d>5$ \\
\hline$r / \%$ day $^{-1}$ & 1.7 & 0.5 & 0.2 & 0.1
\end{tabular}

Table 2: The modelled growth rate designated according to the approximate distance, $d$, from the reserve boundary.

\subsection{The Diffusion Coefficient, $\lambda$}

No information, whatsoever, would appear to be available on the diffusion rates of either $G$. brevipalpis or G. austeni. The most comprehensive set of measurements are probably those for G. morsitans, recorded by JACKSON [17] (reported in ROGERS [24]). The rate at which $G$. morsitans dispersed in G. swynnertoni habitat was found to be a consistant $0.153 \mathrm{~km}^{2} \mathrm{day}^{-1}$, so long as the initial stages of the experiment are omitted. Other work, mostly by the same author (also summarised in RoGERs [24]), suggests G. morsitans was possibly uncomfortable in $G$. swynnertoni habitat. The very low end of the G. morsitans range would appear to be about $0.04 \mathrm{~km}^{2} \mathrm{day}^{-1}$. The point is that different habitats can have different coefficients, as do different species, and one would imagine temperature plays a role. G. brevipalpis and G. austeni are profoundly different species to G. morsitans, in both size and habitat. G. morsitans is of an intermediate size, while G. brevipalpis is one of the largest flies known. G. austeni is the smallest of the tsetse flies. G. brevipalpis and G. austeni are both forest-dwelling, whereas G. morsitans is a savannah species.

The lack of known parameters need not necessarily be cause for despair. Worst-case values are sought and such values are readily deduced from Figure 2. The premise of this work is that the reserve is a problem, that it is the cause of unusually high tsetse numbers in the adjacent agricultural areas. Indeed, the difference in habitat, visibly discernable in satellite images, and Figure 2 are certainly suggestive of a haven with a zone of influence, in the case of a very habitat-specific G. brevipalpis. The distribution of G. austeni is less well understood as there appear to be areas of good $G$. austeni habitat outside the reserve. It is arguable whether an analogous, likely zone of influence can be detected about the reserve; that is until one casts one's eyes on the St. Lucia region, to the east, for corroboration.

Worst-case diffusion coefficients for both G. brevipalpis and G. austeni were revealed by a strategy of trial and error. Different values were used until matching zones of influence to those evidenced by Figure 2 were produced. The value of the diffusion coefficient was either halved, or doubled, until a suitable zone of influence was generated. The transition through 

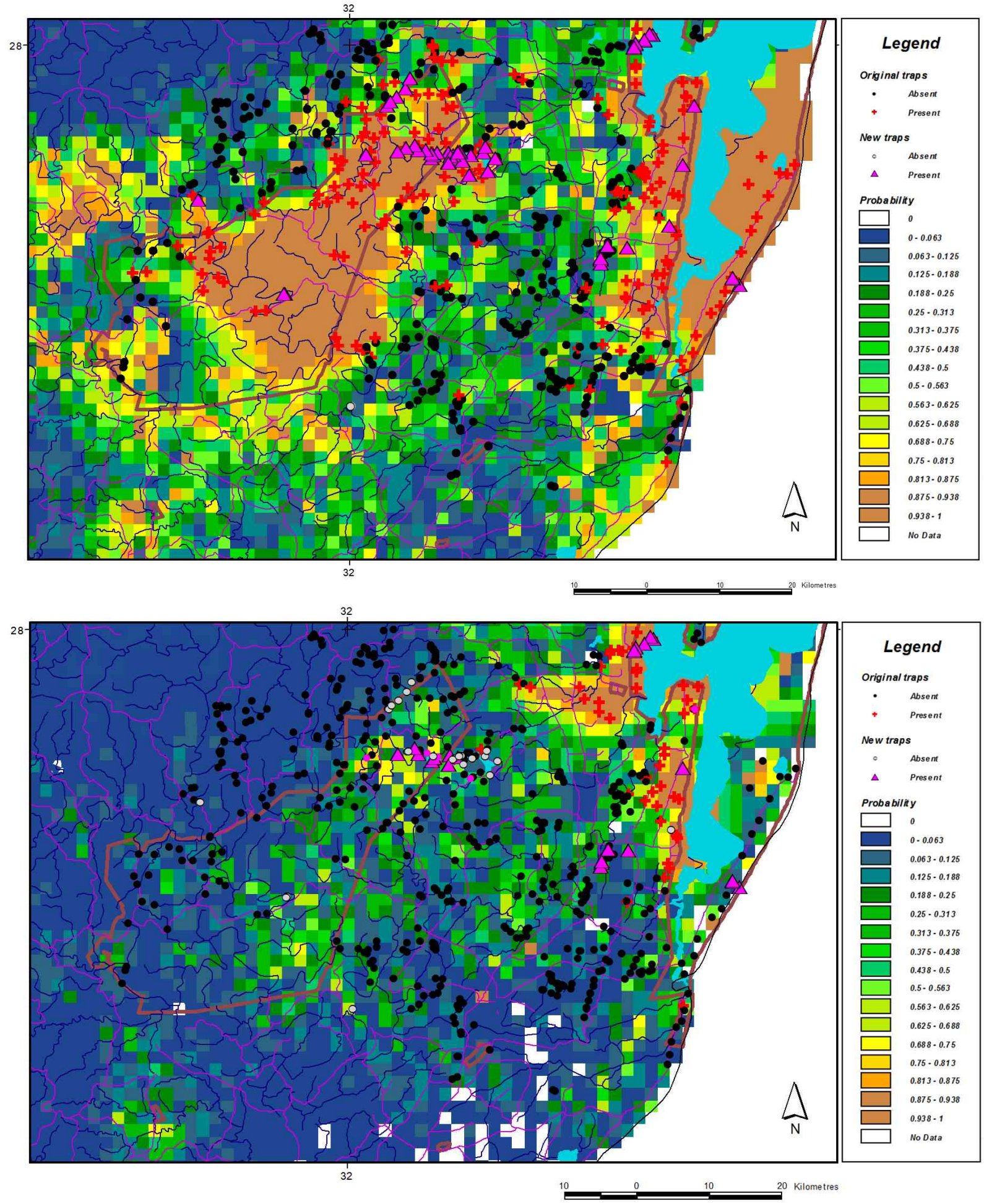

Figure 2: G. brevipalpis risk (top) and G. austeni risk (bottom). Source: HENDRICKX [16]. 


\section{Candidates for the Worst-Case Diffusion Rates of $G$. austeni and G. brevipalpis}
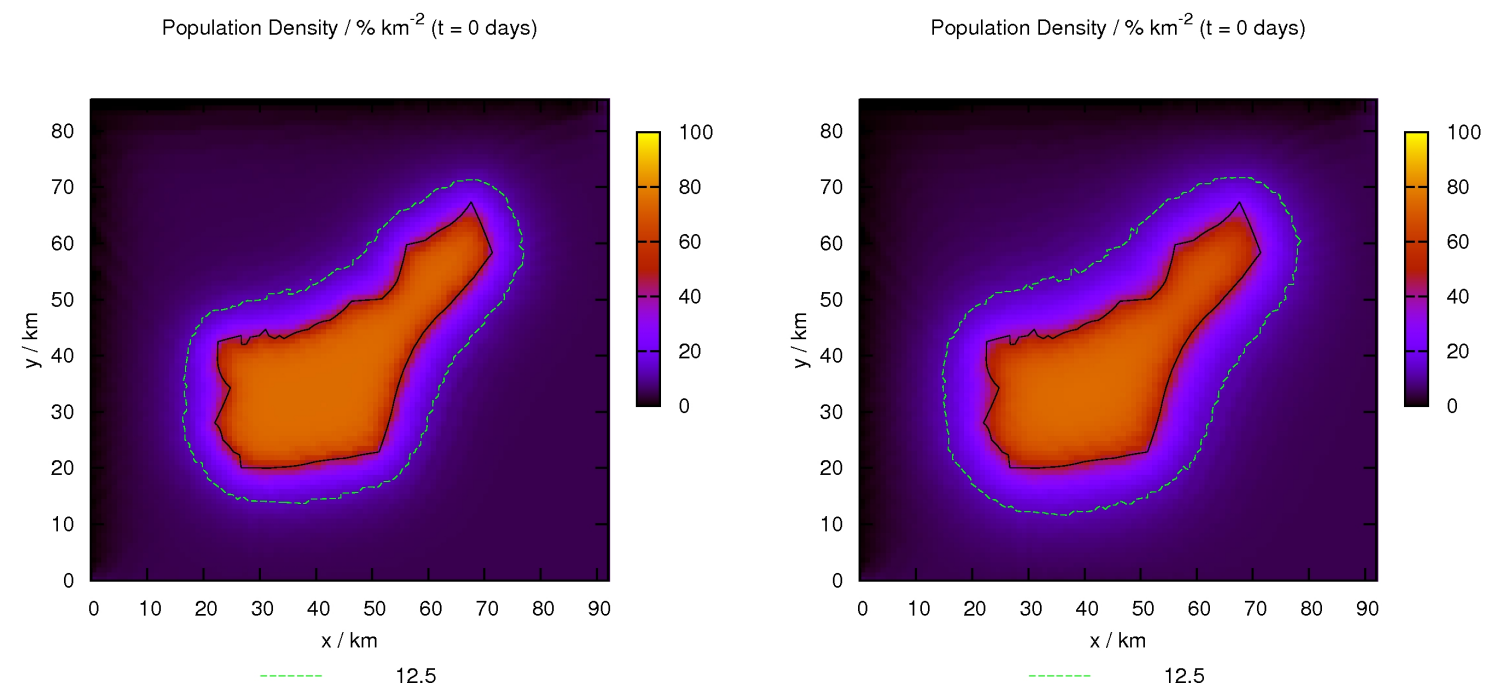

Figure 3: Computed steady states for $\lambda=0.04 \mathrm{~km}^{2}$ day $^{-1}$ (at left) and $\lambda=0.08 \mathrm{~km}^{2}$ day $^{-1}$ (at right) using $G$. austeni carrying capacities.
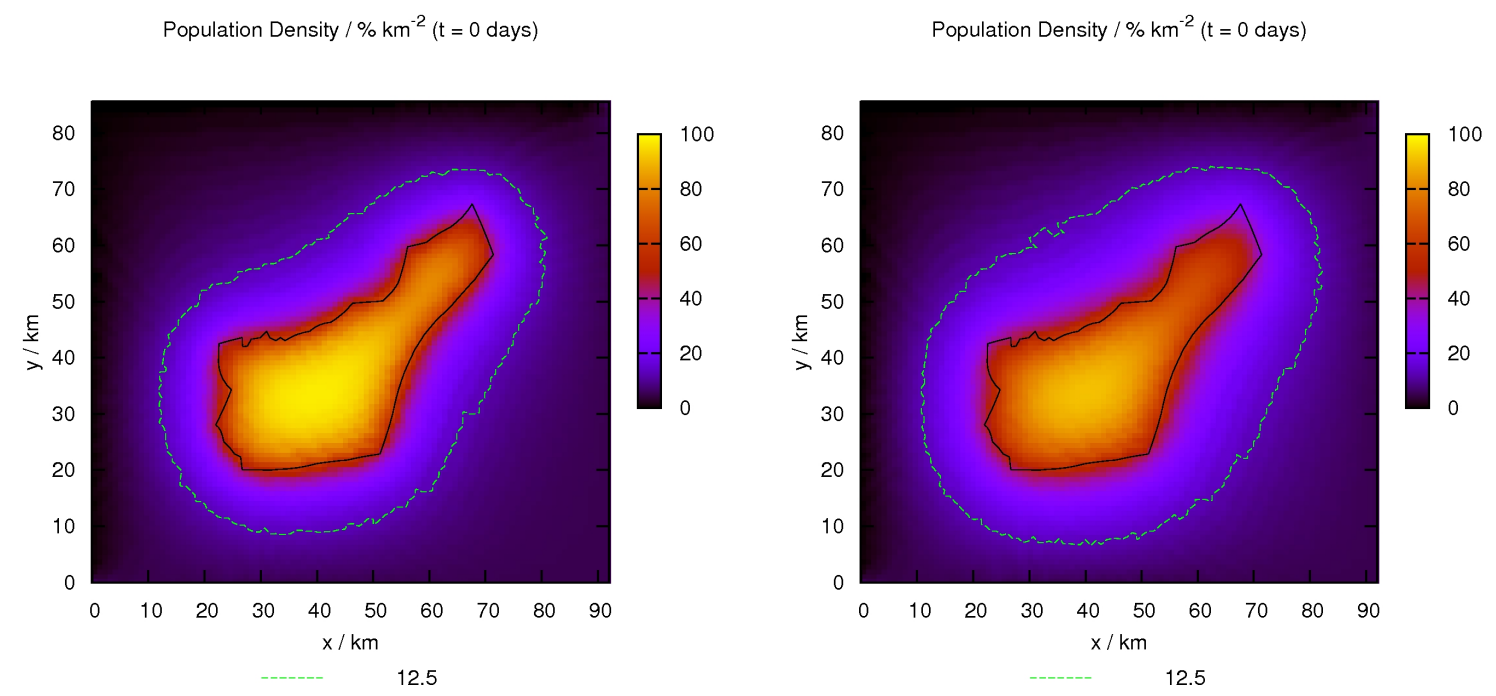

Figure 4: Computed steady states for $\lambda=0.16 \mathrm{~km}^{2}$ day $^{-1}$ (at left) and $\lambda=0.32 \mathrm{~km}^{2} \mathrm{day}^{-1}$ (at right) using G. brevipalpis carrying capacities. Notice that the boundary conditions are starting to effect the computed zone of influence at these high diffusion rates. 
$12.5 \%$ was deemed the most distinct in the G. brevipalpis data. The final matches, Figures 3 and 4, were obviously not perfect due to higher ground to the north, suitable habitat outside the reserve (which was not modelled) and supplementation from the St. Lucia side of the reserve. With hindsight, a bigger domain would have been preferred. The crude technique, nonetheless, suggested some very acceptable values.

The diffusion coefficient for G. austeni is probably $0.04 \mathrm{~km}^{2}$ day ${ }^{-1}$, that of a very comfortable G. morsitans population. Intuitively, this makes sense because of the small size of the species. At worst, one might speculate that it could reach $0.08 \mathrm{~km}^{2}$ day $^{-1}$. The diffusion coefficient for $G$. brevipalpis, however, came as something of a surprise for a forest-dwelling species. At somewhere between $0.16 \mathrm{~km}^{2} \mathrm{day}^{-1}$ and, very possibly as high as, $0.32 \mathrm{~km}^{2} \mathrm{day}^{-1}$, it approaches ROGERS [24]'s observations of G. fuscipes fuscipes, a fly of similar habitat, though smaller size.

\subsection{The Temperature, $T$}

The South African Meteorological Services quote a mean annual temperature of $22.1{ }^{\circ} \mathrm{C}$ for Mpila, inside the reserve (based on data collected during the 1980's and 1990's). This is consistant with the data of SCHULZE and MAHARAJ [26], who define the overall area as being of a temperature greater than $22{ }^{\circ} \mathrm{C}$. The Mpila value is further corroborated by ARC-ISCW automatic weather stations situated between Mtunzini and Pongola (operational since 2004). They suggest an average daily temperature of $22^{\circ} \mathrm{C}$, according to the Department of Agriculture. The daily temperature for the entire region was taken to be the mean annual temperature of Mpila, $22.1^{\circ} \mathrm{C}$.

\subsection{The Finite Element Mesh}

Nine-noded quadrilateral elements and the associated $Q_{2}$ element basis were used. A program to generate the mesh was written based on CHILDS and REDDY [5]. 


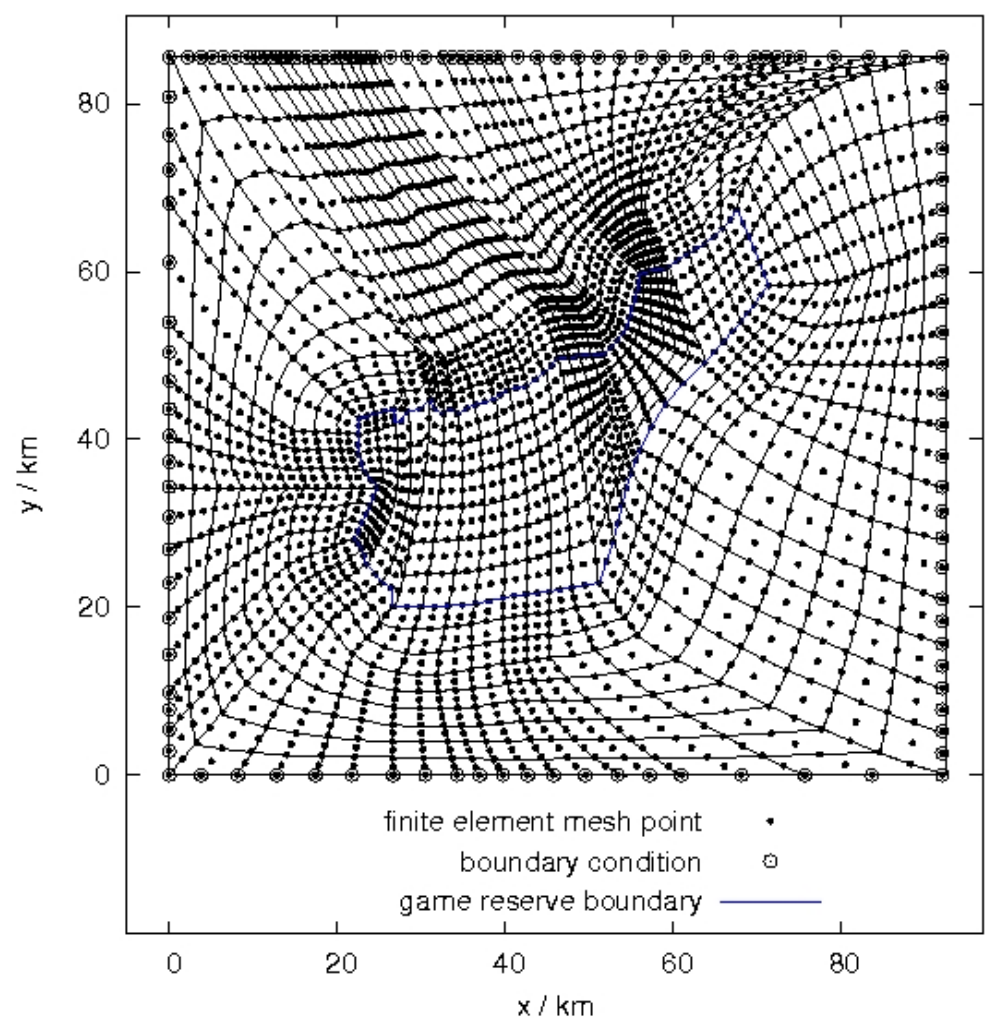

Figure 5: The finite element mesh.

\subsection{Boundary Conditions}

The population was assumed to vanish along the northern and western boundaries. A constant supply of flies, maintaining the eastern and southern boundaries of the entire region at a 5\% level, was assumed.

\section{Simulation}

The topic of simulation has already been broached in determining diffusion coefficients. The simulations attempted to address the following questions, which were identified as relevant to the Hluhluwe-iMfolozi problem: 
1. What are the likely, worst-case diffusion coefficients for G. brevipalpis and G. austeni? (Completed in Section 5.3)

2. What will the long-term effect of the temporary, 2-year use of pour-ons in the surrounding areas be?

3. Can the populations within the reserve be 'siphoned out' to extinction from outside the reserve; failing that, down to the $20 \%$ level?

4. What is a practical barrier width?

5. Can the influence of the Hluhluwe-iMfolozi Game Reserve on surrounding tsetse population levels be neutralized?

6. Can the tsetse populations of the Hluhluwe-iMfolozi Game Reserve and their associated trypanosomiasis be isolated from the surrounding areas?

7. In what way are diffusion rates relevant to containment, eradication and any subsequent rebound?

The initial, start-up values were taken to be the carrying capacities, with the exception of the northern and western boundaries, where the population was assumed to vanish. For each simulation the model was first run for two years; more than adequate time to allow it to settle down to the steady-state from its start-up values. The model was then run for another two years ${ }^{1}$ with various controls in place. In instances in which the controls were either revised or removed, the population was allowed to rebound for a further one or two years. Unless otherwise stated, $G$. austeni carrying capacities were used in association with diffusion rates of $0.08 \mathrm{~km}^{2} \mathrm{day}^{-1}$ and below, while $G$. brevipalpis carrying capacities were used in association with diffusion rates of $0.16 \mathrm{~km}^{2}$ day $^{-1}$ and above.

Barriers of a width greater than $5 \mathrm{~km}$ were not experimented with, even though they can be expected to be more optimal in terms of the required number of targets. This is since they were deemed to be a self-defeating waste of land. The barriers were modelled in such a way that their quoted widths usually included a reasonable safety margin. This built-in, safety margin was increased substantially $(\approx-15 \%)$ along the northern, concave boundary of the reserve, for the purposes of a crude sensitivity analysis. Incorrect barrier construction, theft, storms, malfunction and fires (such as are visible in Figure 1) are all eventualities which must be prepared for. A reduction of the Figure 3 and 4 tsetse levels by an order of magnitude was adopted as a guideline for the tolerance used in evaluating barriers. (It is reasoned that if the incidence of nagana due to flies from the game reserve was $10 \%$ for a given time frame, a barrier with such a tolerance should cause it to drop below $1 \%$.) Thus, a zone with a population density lower than $4 \% \mathrm{~km}^{-2}$ is considered to be substantially vacant. No flies are able to leave a vacant zone, which, in turn, means no flies ever cross one of the size in question. The value of $4 \% \mathrm{~km}^{-2}$ is also in keeping with HENDRICKX [16]'s lowest detectable level, the $0 \%$ to $6.3 \%$ category.

\footnotetext{
${ }^{1}$ Deltamethrin pour-ons can not safely be used on cattle for any longer than two years without compromising their resistance to tick-bourne diseases, consequently an enzootic condition.
} 


\subsection{Results}

\section{A $2.5 \mathrm{~km}$-Wide Versus a $5 \mathrm{~km}$-Wide Barrier Surrounding the Reserve}
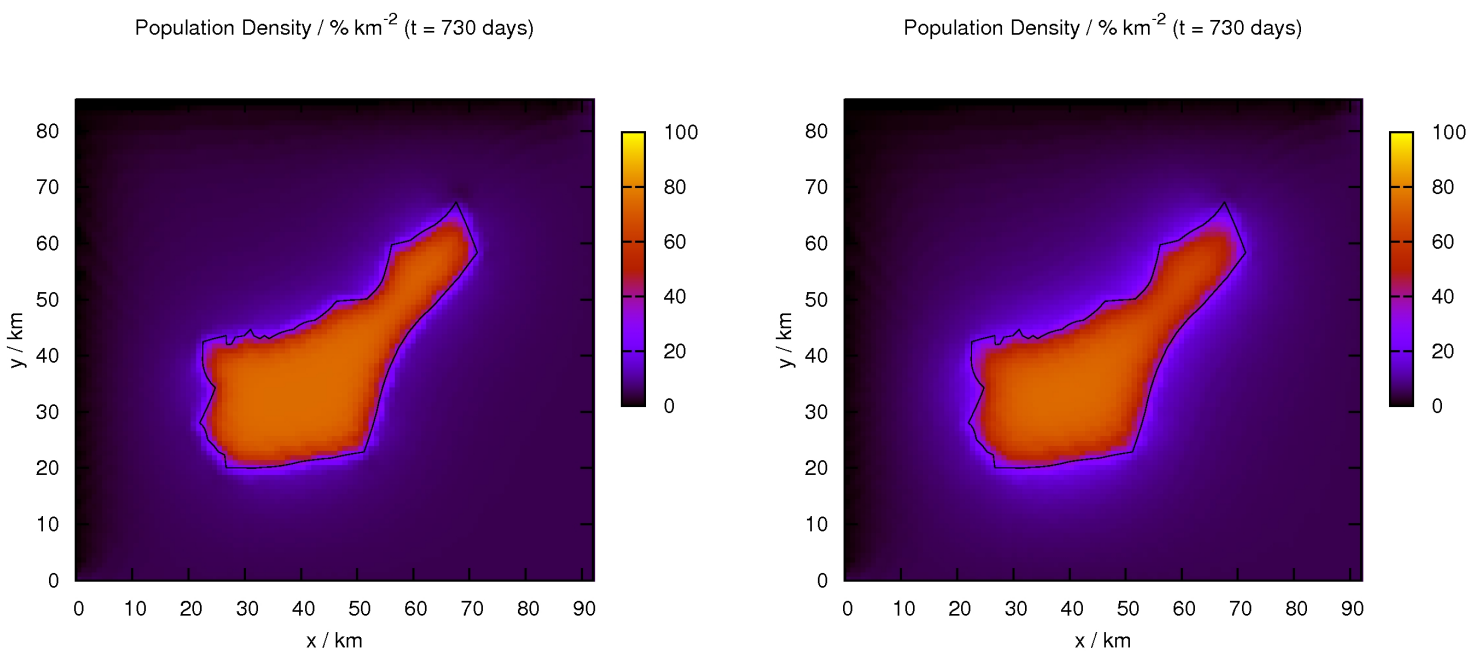

Figure 6: The effect of an approximately $2.5 \mathrm{~km}$-wide barrier, with a $2 \%$ day $^{-1}$ mortality throughout, after 2 years. At left, $\lambda=0.04 \mathrm{~km}^{2}$ day $^{-1}$. At right, $\lambda=0.08 \mathrm{~km}^{2} \mathrm{day}^{-1}$. A $2.5 \mathrm{~km}$-wide barrier is clearly not efficacious.
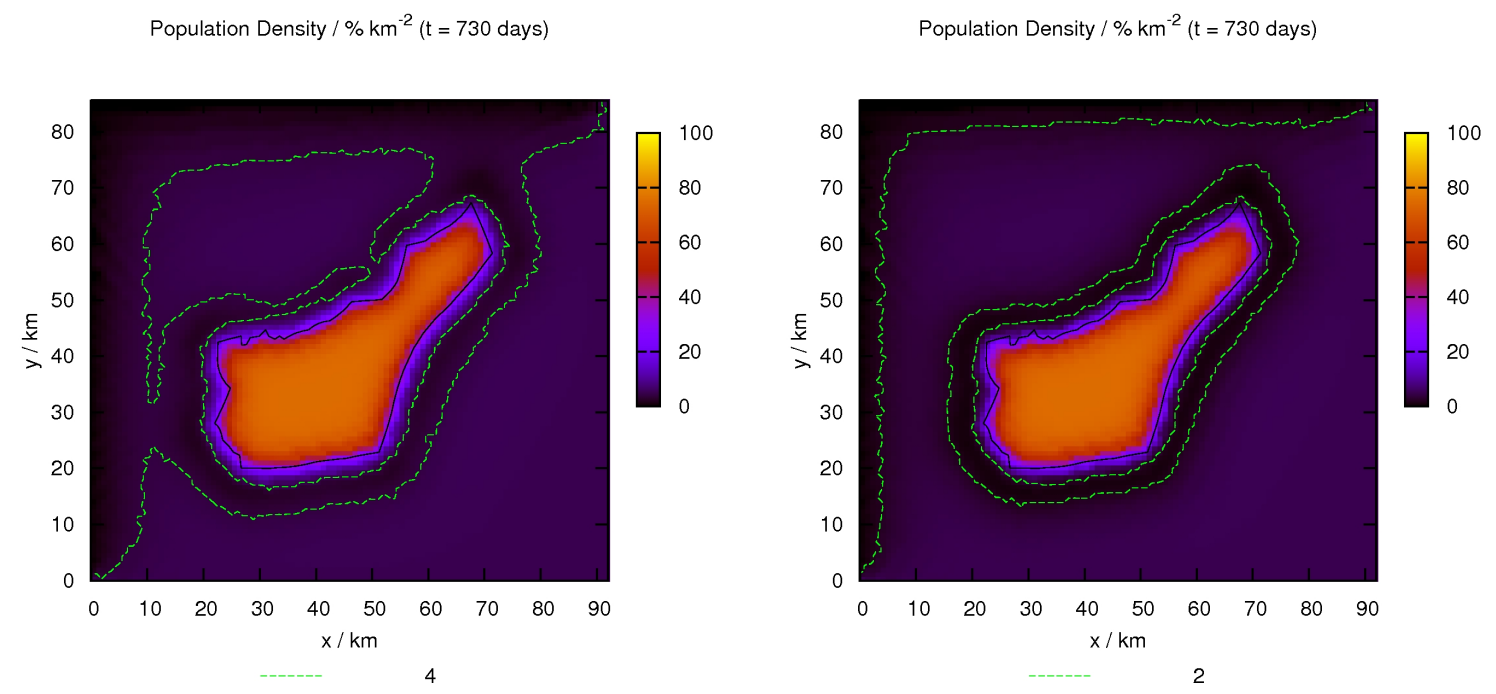

Figure 7: The effect of an approximately $5 \mathrm{~km}$-wide barrier, after 2 years, using $\lambda=0.04 \mathrm{~km}^{2}$ day $^{-1}$. At left, a barrier with a mortality of $2 \%$ day $^{-1}$ throughout. At right, a barrier with a mortality of $4 \%$ day $^{-1}$ throughout. The $5 \mathrm{~km}$-wide barrier has already successfully isolated the G. austeni, reserve population, should it have a diffusion rate as low as $0.04 \mathrm{~km}^{2}$ day $^{-1}$. 


\section{The Quest for an Impenetrable, Surrounding Barrier}
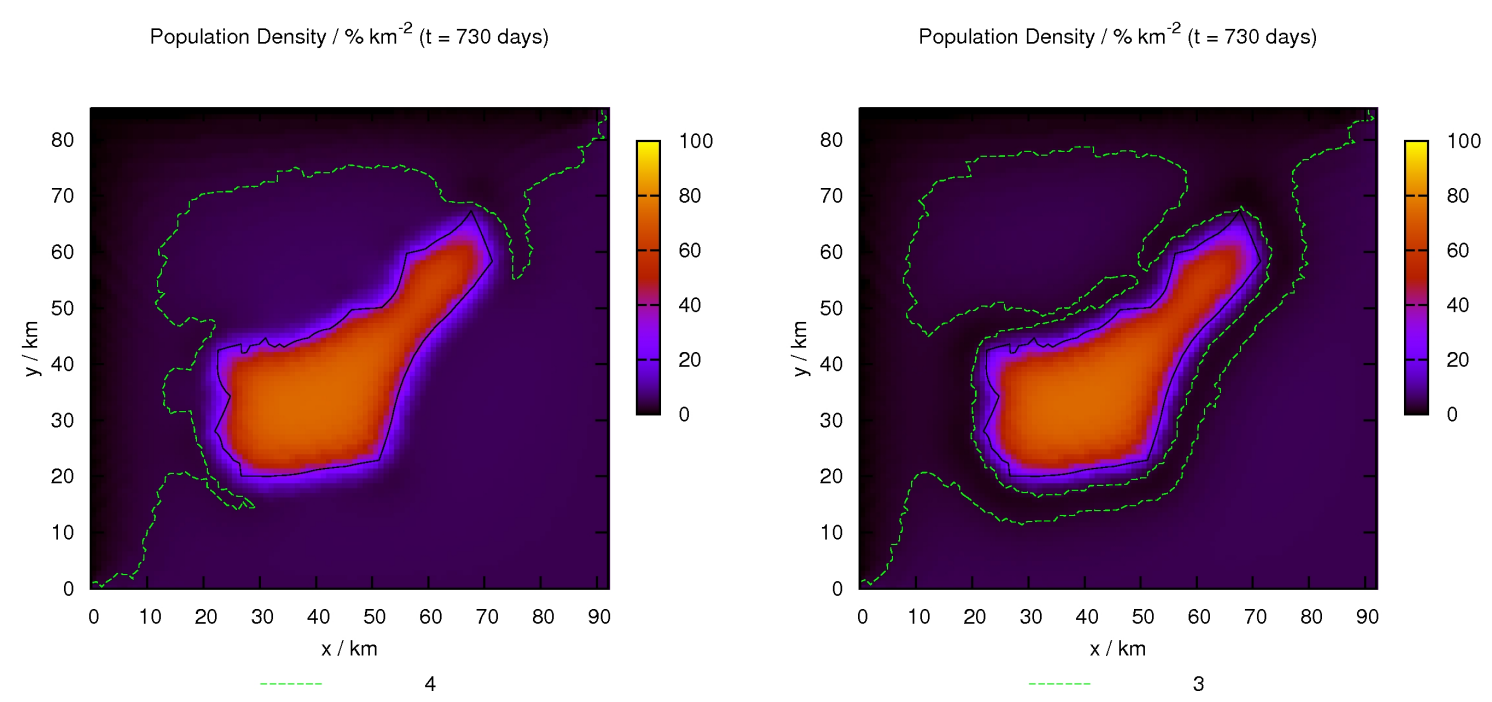

Figure 8: The effect of an approximately $5 \mathrm{~km}$-wide barrier, after 2 years, using $\lambda=0.08 \mathrm{~km}^{2}$ day $^{-1}$. At left, a $2 \%$ day $^{-1}$ mortality throughout the barrier neutralizes the influence of the reserve on surrounding tsetse populations, however, flies with a greater prevalence and more lethal strains of trypanosomiasis are still able to commute. At right, using a $4 \%$ day $^{-1}$ mortality throughout, the barrier has isolated the reserve.
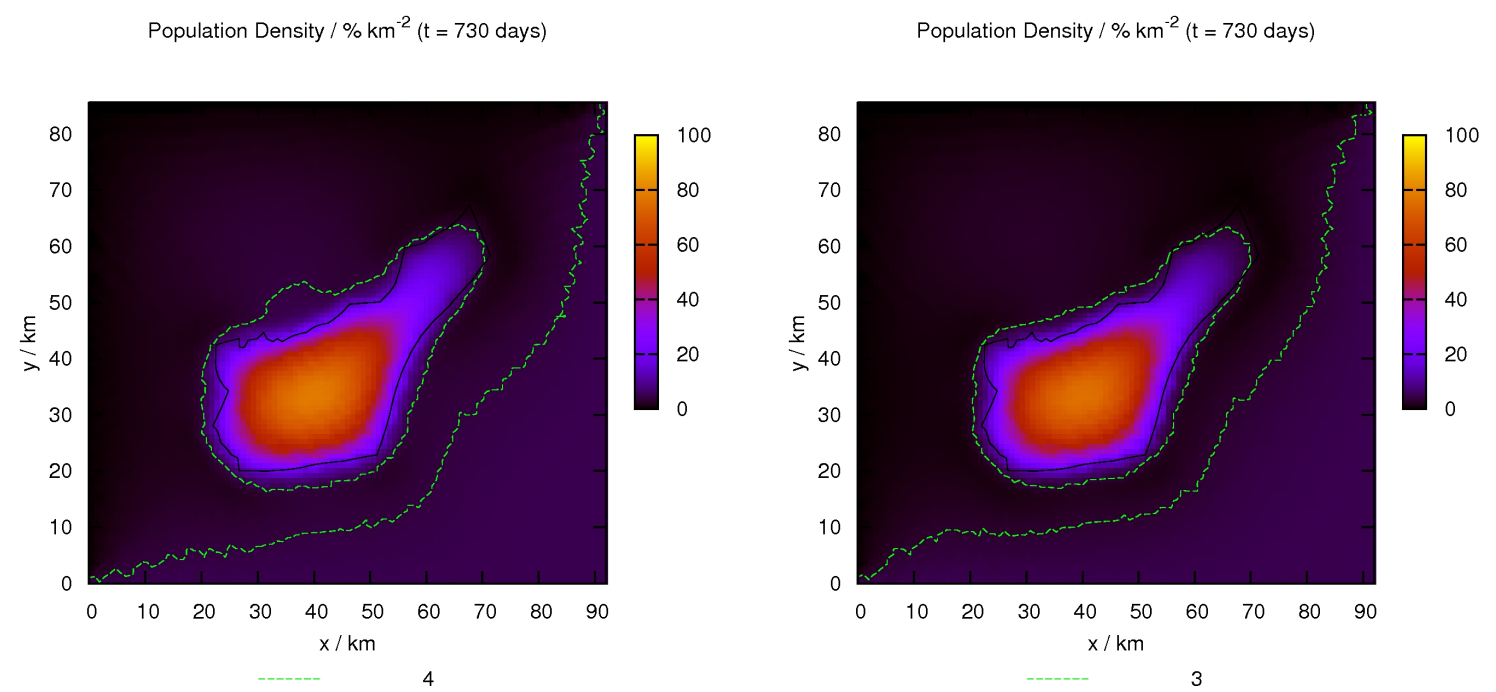

Figure 9: The effect of an approximately $5 \mathrm{~km}$-wide barrier, after 2 years, using $\lambda=0.32 \mathrm{~km}^{2} \mathrm{day}^{-1}$. At left, an $8 \%$ day $^{-1}$ mortality throughout the barrier just fails to isolate the reserve and then only along that part of the barrier-zone constructed to be $15 \%$ narrower for the purposes of sensitivity analysis. At right, using a $12 \%$ day $^{-1}$ mortality throughout, the barrier has isolated the reserve. 


\section{Pour-Ons and the Subsequent Rebound}
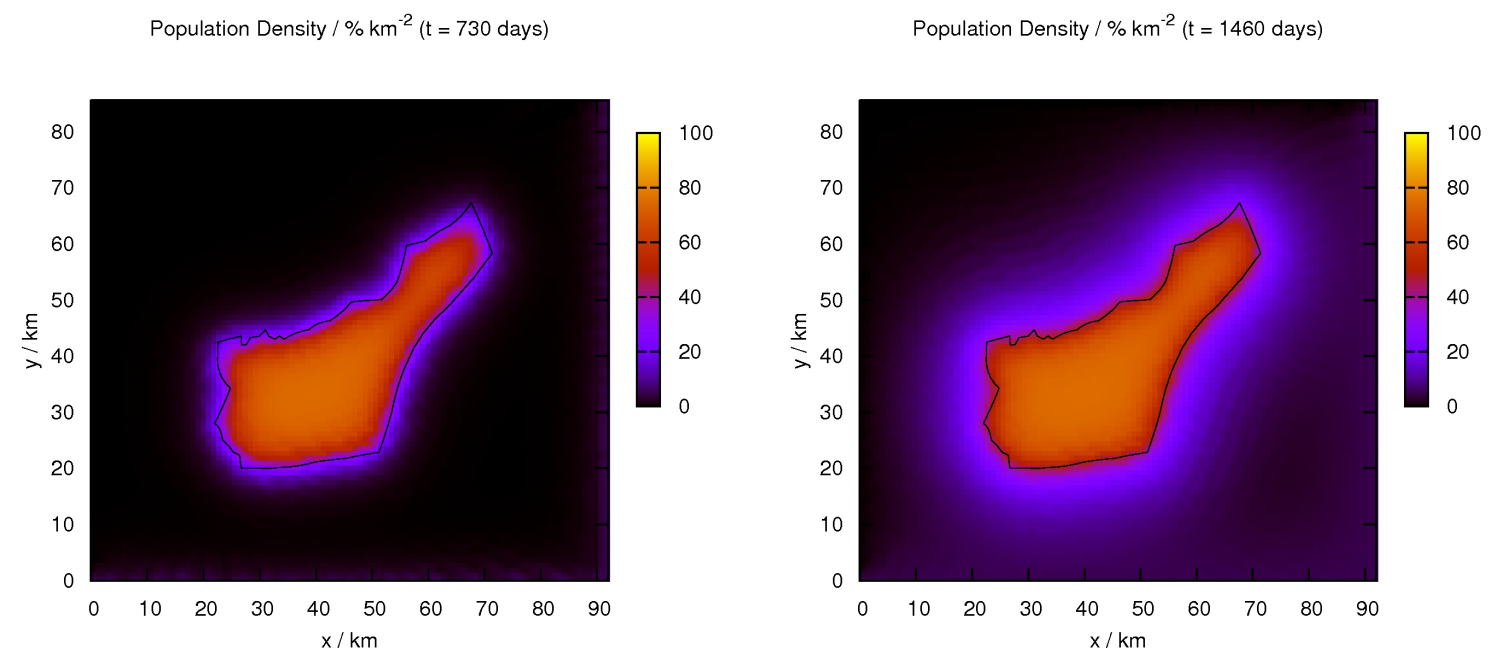

Figure 10: The effect of a $2 \%$ day $^{-1}$ mortality imposed everywhere outside the reserve, for a period of 2 years, using $\lambda=0.08 \mathrm{~km}^{2}$ day $^{-1}$ (left); the rebound after a further 2 years (right). There is a complete rebound into the areas immediately surrounding the reserve, however, more remote areas of poor habitat do not recover as quickly.

Population Density $/ \% \mathrm{~km}^{-2}(\mathrm{t}=730$ days $)$

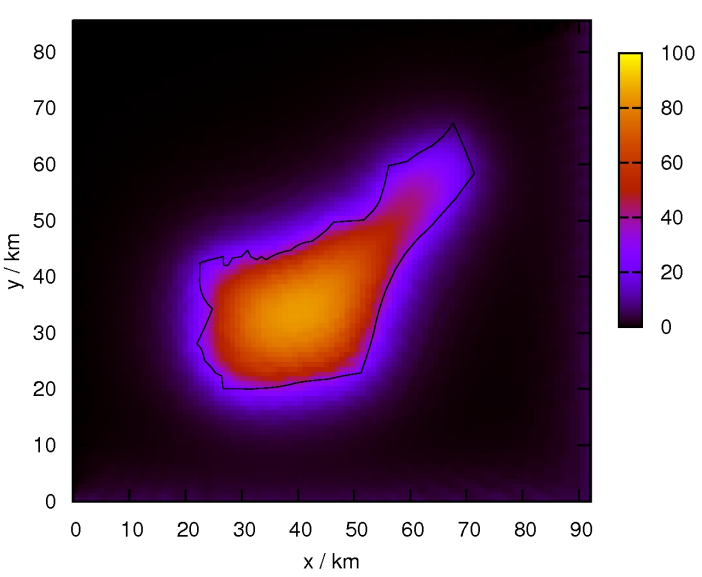

Population Density $/ \% \mathrm{~km}^{-2}(\mathrm{t}=1460$ days $)$

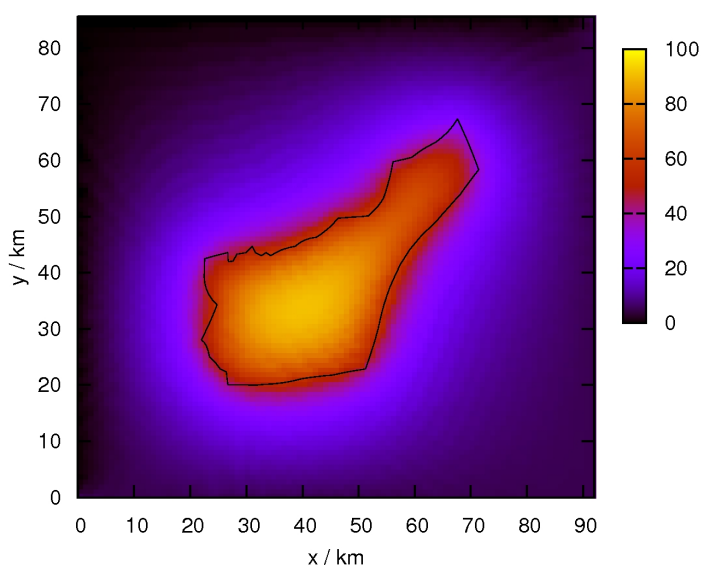

Figure 11: The effect of a $2 \%$ day $^{-1}$ mortality imposed everywhere outside the reserve, for a period of 2 years, using $\lambda=0.32 \mathrm{~km}^{2}$ day $^{-1}$ (left); the rebound after a further 2 years (right). There is a complete rebound. 
The Implications of the Diffusion Coefficient for Eradication and Any Subsequent Rebound
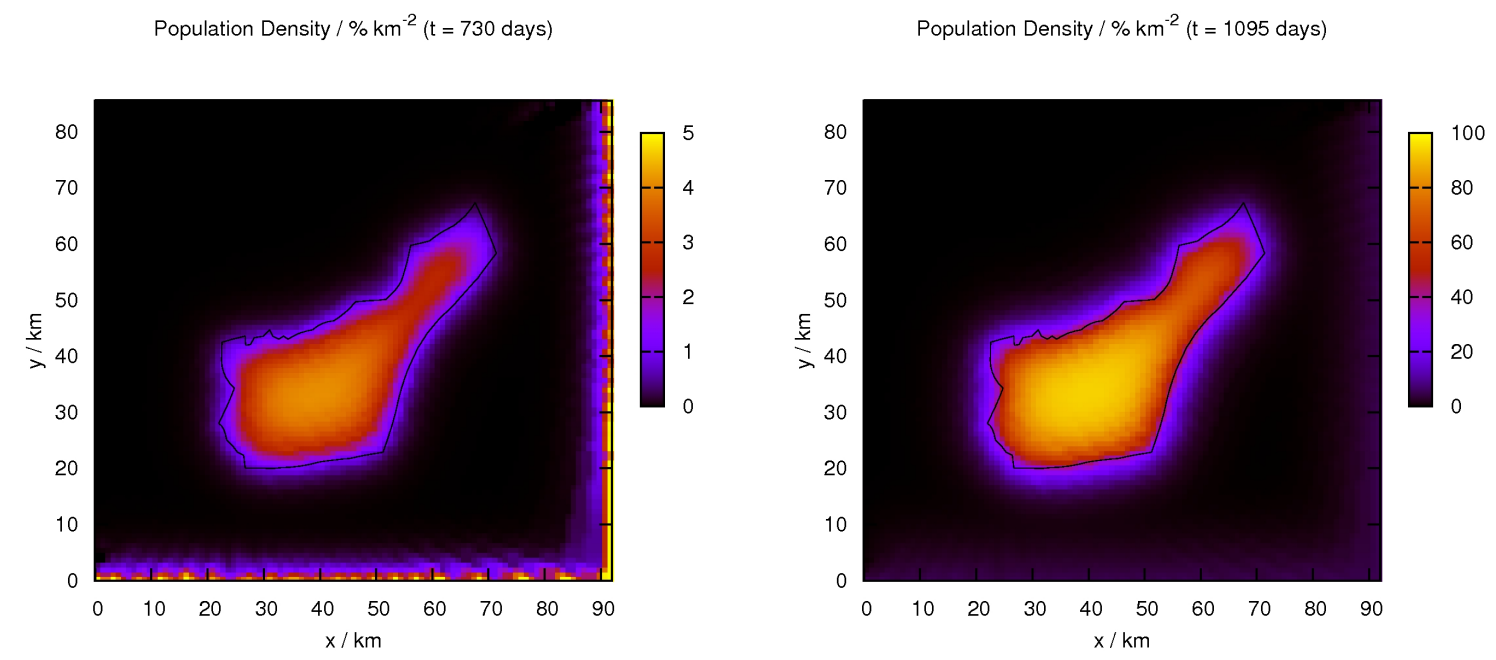

Figure 12: The effect of a $2 \%$ day $^{-1}$ mortality imposed everywhere for a period of 2 years, using $\lambda=0.04 \mathrm{~km}^{2}$ day $^{-1}$ (left); the population rebound after a further year (right). $G$. brevipalpis carrying capacities were used for comparative purposes.
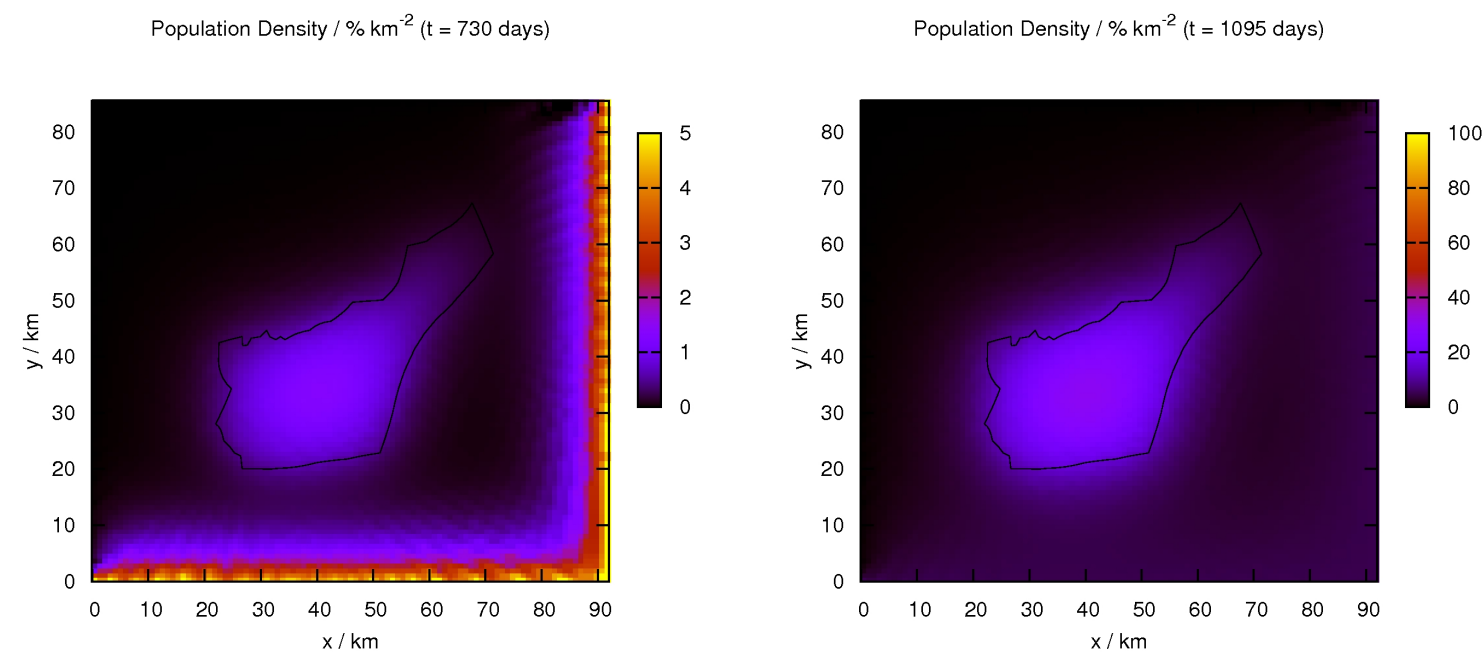

Figure 13: The effect of a $2 \%$ day $^{-1}$ mortality imposed everywhere for a period of 2 years, using $\lambda=0.32 \mathrm{~km}^{2}$ day $^{-1}$ (left); the population rebound after a further year (right). More mobile species are more vulnerable to targets and make a slower recovery, all other things being equal. 
'Siphoning' Out the Reserve Population from the Boundary

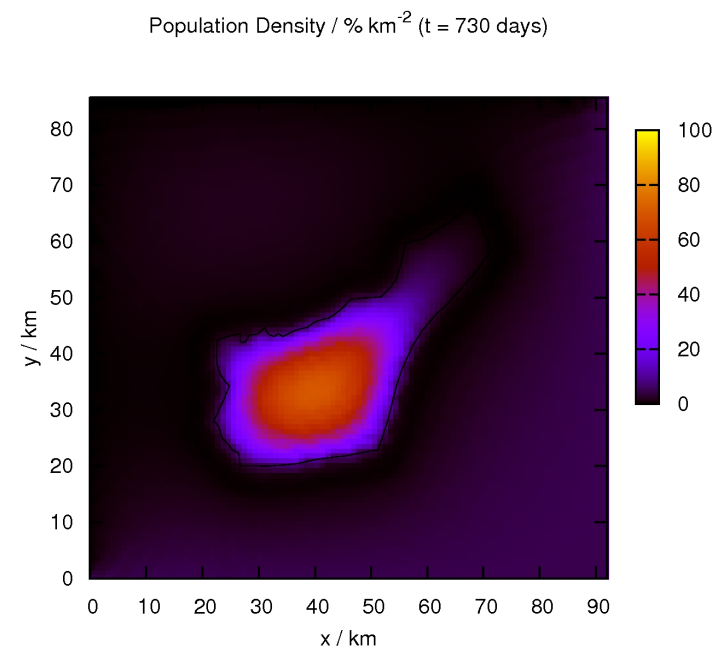

Figure 14: The effect of a $5 \mathrm{~km}$-wide barrier in which a $50 \%$ day $^{-1}$ mortality is imposed throughout, for a period of 2 years, using $\lambda=0.32 \mathrm{~km}^{2}$ day ${ }^{-1}$. The reserve is $960 \mathrm{~km}^{2}$ and its tsetse populations cannot be removed from the boundary using current target technology.

\section{Improvising Barriers According to Specified Mortality Rates}

Odour-baited targets and cattle treated with so-called 'pour-ons' are the means by which tsetse barriers can be constructed.

\subsection{Odour-Baited Targets (and the Possible Revelation of Competition)}

The definitive experimental work involving target barriers for G. austeni and G. brevipalpis is that of Esterhuizen, Kappmeier Green, Nevill and Van Den Bossche [7]. Essentially they barricaded a small peninsula with targets. They also placed targets on the peninsula itself and measured the decline of G. austeni and G. brevipalpis in relation to the target density on the peninsula. At a target density of $8 \mathrm{~km}^{-2} G$. austeni was found to decline at a growth rate of around -0.014 day $^{-1}$.

The translation of such a growth rate into target mortality is facilitated by WILLIAMS, DRANSFIELD and BRIGHTWELL [30]'s seminal equation. In their equation

$$
\beta e^{-\tau_{0}\left(\delta_{0}+R\right)-\tau_{1}\left(\delta_{1}+R\right)-\tau_{2}\left(\delta_{2}+R\right)}=1-e^{-\tau_{2}\left(\delta_{2}+R\right)},
$$

$R$ is the growth rate, $\beta$ is the fecundity, the $\delta_{i}$ are mortalities, the $\tau_{i}$ are durations and the subscripts 0,1 , and 2 pertain to the puparial, pre-ovulatory and interlarval stages respectively. 
Despite good estimates of the rate of $G$. austeni decline, choosing other parameters in the equation is something of a heuristic exercise. If one takes cognizance of ROGERS and RANDOLPH [25]'s findings on predation, the pupal water loss model of CHILDS [4] etc., a $2 \%$ day $^{-1}$ natural mortality rate among pupae would not be unreasonable for the species in question. Natural mortality for the pre-ovulatory cohort is known to be high. It includes massive teneral mortality and although it does not fall victim to targets in the same proportions as the adults, some still do (HARGROVE [13]). With this fact in mind the pre-ovulatory stage flies were assigned a natural mortality of $2.2 \%$ day $^{-1}$ and assumed to have a target-related mortality one half that of adults. At $22.1{ }^{\circ} \mathrm{C}$ the relevant formulae ${ }^{1}$ for the first and subsequent interlarval periods predict 17.5 days and 10.5 days respectively. The formula Equation 2 gives a puparial duration of 34.6 days for females. A miscarriage rate of 5\%, and therefore a fecundity of 0.475 was used (in keeping with WILliams ET AL. [30]). Solving Equation 3, using Newton's method and assuming a pre-existing equilibrium involving the aforementioned parameters, suggested a natural mortality of $1.77 \%$ day $^{-1}$ among the adults.

Solving Equation 3, using Newton's method and the newly completed set of parameters, suggested the $8 \mathrm{~km}^{-2}$ target density of ESTERHUIZEN ET. AL. [7] was equivalent to an artificially imposed mortality of $2.39 \%$ day $^{-1}\left(0.30 \%\right.$ day $^{-1}$ per target $)$. Target-related mortality is obviously much lower for these forest species. In comparison, a single odour-baited target ${ }^{2}$ kills $2 \%$ day $^{-1}$ of the female G. pallidipes population (HARGROVE [11]).

REMARK: Note that a value of $2.39 \%$ day $^{-1}$ is in very close agreement with the modelled, $2 \%$ day $^{-1}$ barrier-mortality required for the isolation of $G$. austeni, as are the widths of the modelled barrier and ESTERHUIZEN ET AL. [7]'s peninsula barricade respectively. The work of ESTERHUIZEN ET AL. [7] was only subsequently brought to the author's attention.

So far as G. brevipalpis is concerned, ESTERHUIZEN ET AL. [7]'s results are not as clear. Indeed, the results of this work suggest their barrier-zone might have been completely ineffectual against a very mobile G. brevipalpis. Another possibility is that G. brevipalpis is completely impartial to odour-baited targets. Yet something certainly did happen in both target-containing sectors when the concentration of targets reached a density of $8 \mathrm{~km}^{-2}$. G. brevipalpis initially declined at a rate indicative of an imposed mortality of $0.63 \%$ day $^{-1}$ (using Equation 3, again). A subsequent reversal of this decline then coincided with the demise of $G$. austeni and the $G$. brevipalpis population grew to levels never previously attained; in spite of the targets. A number of explanations spring to mind. One argument is that the data is too poor, that what is being observed is simply random noise, should be ignored. Another possibility is that there was a delay in recolonization by this highly mobile species. Certainly one has good reason to suspect an element of diffusion to be operative, even if not the overriding analysis. Why then, the delay? There could be reasons.

An alternative explanation is that the reversal in fortune of the one species coincided with the demise of the other due to the two being in competition: So deleterious was the presence of $G$. austeni to G. brevipalpis that its removal is able to counteract the imposition of a $0.63 \%$ day $^{-1}$ mortality on $G$. brevipalpis (a decline in growth rate of -0.0039 day $^{-1}$ ). In retrospect,

\footnotetext{
${ }^{1}$ HARGROVE [12]'s improved EAST AFRICAN High COMMISION [1] formulae.

${ }^{2}$ As specified in Vale, Hargrove, Cockbill and Phelps [28].
} 
such a situation might have been anticipated. Indeed, one of the posits of this model is that the limitations on growth at pupal sites are density dependent. Pupal habitat for G. brevipalpis is more stringently confined than for G. austeni (according to demonstrations of the pupal water loss model in CHILDS [4]) and the G. austeni puparial duration is a full $20 \%$ shorter than that of $G$. brevipalpis (PARKER [21]). One would imagine $G$. brevipalpis also has an adverse effect on G. austeni. Just how severe and whether or not it can be exploited, is not evident. Further experimentation is required. That ESTERHUIZEN ET AL. [7] were simply not able to measure a true value for the target-related mortality of $G$. brevipalpis, owing to high diffusion rates, is their own conclusion.

If the accepted wisdom is correct that the effect of uniformly distributed targets is additive, then a given mortality may be designed in terms of Table 3 as follows.

\begin{tabular}{l|cccc}
$\delta /$ day $^{-1}$ & 0.02 & 0.04 & 0.08 & 0.12 \\
\hline G. austeni & 7 & 13 & 27 & 40 \\
G. brevipalpis & 25 & 51 & 102 & 152 \\
G. pallidipes & 1 & 2 & 4 & 6
\end{tabular}

Table 3: The number of targets per $\mathrm{km}^{2}$ which will produce a required daily mortality, $\delta$, for each species.

\subsection{Tethered, Treated Cattle}

Unpublished experiments by S. J. Torr (reported in HARGROVE, TORR and KINDNESS [15]) suggest that a single odour-baited target kills the equivalent number of $G$. pallidipes females in $1 \mathrm{~km}^{2}$ as an insecticide-treated ox of weight $400 \mathrm{~kg}$ does in a day. Since ESTERHUIZEN ET AL. [7] used the same $1.5 \times 1 \mathrm{~m}$, black-blue-black targets (manufactured by Bonar Industries, Harare $)^{1}$, the corresponding target-related mortality should apply to the ox for G. austeni and $G$. brevipalpis; assuming these species do not discriminate any differently between the chemical signatures of the beast and the target.

\footnotetext{
${ }^{1}$ ESTERHUIZEN [6] and HARGROVE [13]
} 


\subsection{Treated Herds}

In Hargrove, Holloway, Vale, Gough and Hall [14] it was determined that tsetse catches changed with the tonnage of cattle, $m$, in a ventilated shed and could be described by

$$
\delta \propto 4 m^{0.475} .
$$

Torr's experiment (reported in HARGROVE ET AL. [15]) allows the constants in the simplistic model,

$$
\left[\begin{array}{c}
0.0030 \\
0.00079 \\
0.02
\end{array}\right]=4\left[\begin{array}{c}
c_{\text {austeni }} \\
c_{\text {brevipalpis }} \\
c_{\text {pallidipes }}
\end{array}\right] 0.4^{0.475},
$$

to be determined. The minimum tonnage of cattle required to induce a given daily mortality in a square kilometre is therefore given by Table 4.

\begin{tabular}{c|ccc} 
species & G. austeni & G. brevipalpis & G. pallidipes \\
\hline herd mass / tons $\mathrm{km}^{-2}$ & $0.4\left(\frac{\delta}{0.0030}\right)^{\frac{1}{0.475}}$ & $0.4\left(\frac{\delta}{0.00079}\right)^{\frac{1}{0.475}}$ & $0.4\left(\frac{\delta}{0.02}\right)^{\frac{1}{0.475}}$
\end{tabular}

Table 4: The treated herd mass required to bring about a given mortality, $\delta$, in each species.

\section{Conclusions}

The diffusion coefficient for G. austeni is probably around $0.04 \mathrm{~km}^{2} \mathrm{day}^{-1}$, although a worstcase value of $0.08 \mathrm{~km}^{2}$ day $^{-1}$ was assumed (Figures 2 and 3). The diffusion coefficient for $G$. brevipalpis can be assumed to be around $0.32 \mathrm{~km}^{2}$ day ${ }^{-1}$, although it could be as low as 0.16 $\mathrm{km}^{2}$ day $^{-1}$ (Figures 2 and 4).

Based on the worst-case values in terms of which the problem was phrased, the simulations suggest that the temporary imposition of a $2 \%$ day $^{-1}$ mortality everywhere outside the reserve for a period of 2 years will have no lasting effect on the influence of the reserve when it comes to either population; although it certainly will eradicate tsetse from areas of poor habitat, outside the reserve (Figures 10 and 11). It is doubtful whether the populations within the reserve can be 'siphoned' or 'pumped out' to extinction, or even the $20 \%$ level, from outside the reserve boundary (Figure 14).

During the initial stages of this work, it became apparent that $2.5 \mathrm{~km}$-wide, target barriers were not efficacious (Figure 6) and their further investigation was abandoned in favour of a $5 \mathrm{~km}$ 
width (Figure 7). The influence of the reserve on surrounding G. austeni population levels can be completely neutralized by a $5 \mathrm{~km}$-wide barrier in which there is a mortality of $2 \%$ day $^{-1}$ throughout (Figure 8). For G. brevipalpis a $5 \mathrm{~km}$ wide barrier to the same end will require a mortality of $8 \%$ day $^{-1}$ throughout (Figure 9). Notice, however, that these measures are not in any way able to address the likelihood of a greater prevalence, as well as more lethal strains, of trypanosome infection in flies close to the reserve boundary, regardless of any reduction in their numbers.

A $5 \mathrm{~km}$-wide barrier of odour-baited targets with a mortality of $4 \%$ day $^{-1}$, throughout, should succeed in isolating a worst-case, Hluhluwe-iMfolozi G. austeni population and its associated trypanosomiasis from the surrounding areas (Figures 7 and 8 ). A more optimistic estimate of its mobility suggests a mortality of $2 \%$ day $^{-1}$ will suffice (Figure 7). These mortalities correspond to a deployment of odour-baited targets ${ }^{1}$ with a minimum density of $13 \mathrm{~km}^{-2}$ and $7 \mathrm{~km}^{-2}$ respectively. Simple arguments suggest that such counter measures should reduce the G. austeni problem associated with the reserve by at least an order of magnitude.

For $G$. brevipalpis, a mortality of $12 \%$ day $^{-1}$, throughout, will achieve the same end of complete isolation (Figure 9). The impartiallity of G. brevipalpis to odour-baited targets is obviously a concern, should this species be conclusively shown to be a vector of trypanosomiasis. A $12 \%$ day $^{-1}$ mortality is not practical in terms of what one can only surmise is the mortality of current odour-baited target technology. A mortality of $8 \%$ day $^{-1}$ fails mainly from the point of view of the crude sensitivity analysis and it will probably suffice if: the width of the barrier can, with absolute certainty, be said to be no less than $5 \mathrm{~km}$; boundaries are non-concave; the context is one involving rebound suppression, following the use of pour-ons for a 2 year period. The less ambitious goal of neutralizing the reserve's influence on the surrounding $G$. brevipalpis population would require a deployment of odour-baited targets with a minimum density of $102 \mathrm{~km}^{-2}$; again a clearly impractical proposition. Any strategy for the control of G. brevipalpis should include the surroundings of the Hluhluwe dam and its backwater, as if it were part of the reserve, based on CHILDS [4].

Extrapolating the work of HARGROVE ET AL. [14] suggests that the substitution of insecticidetreated herds for odour-baited targets is not a viable alternative for the control of the two forest species in question. Required herd-masses are impractical for the purposes of barriers and containment. Individually tetherd, treated cattle can be used as substitutes for odour-baited targets, although the numbers required are probably not really practical either. Periodically rotating them in and out of the barrier zone would prevent a loss of resistance to tick-bourne diseases and an enzootic condition. (Individually tethered, deltamethrin-treated cattle, distributed uniformly throughout a barrier zone, may be less likely than targets to fall victim to the tragedy of the commons type mentality known to prevail among the local population.)

The premise that the entire reserve, and it alone, is a problem is not as valid for G. austeni as it is for G. brevipalpis (Figure 2). In the case of G. austeni it may well be worth singling out individual locii for the application of control measures (e.g. the flood plain of the Hluhluwe River, the vicinities of the Hluhluwe Dam, its backwater and the confluence of the Black Mfolozi and White Mfolozi rivers), based on CHILDS [4] and Figure 2. For G. brevipalpis, however, a

\footnotetext{
${ }^{1}$ As used by Esterhuizen ET AL. [7].
} 
comparison of Figures 2 and 4 suggests that the logistic growth rate might even exceed $1.7 \%$ day $^{-1}$ in the northern, Hluhluwe sector of the reserve. So favourable is that habitat.

For a given mortality, more mobile species are found to be more vulnerable to eradication than more sedentary species while the opposite is true for containment. The scenarios depicted in Figures 12 and 13 demonstrate, firstly, that high diffusion rates are more amenable to eradication since the same target consistancy is not required locally. Species with high diffusion rates are vulnerable to controls which are geographically more remote. Secondly, high diffusion rates lead to a much weaker initial recovery from levels close to extinction ${ }^{2}$. The reason is that there is a tendency to disperse which is not efficacious for logistic growth at low population densities.

One is now presented with a scenario in which G. brevipalpis may be more vulnerable to eradication than containment and vice versa for G. austeni (partiality to odour-baited targets and existing population levels aside). Yet whether or not G. brevipalpis is even an agent of infection is still a moot point (MOTLOANG ET AL. [19]). G. austeni is, in contrast, without the slightest doubt a highly competent vector of trypanosomiasis. The possibility that eliminating G. brevipalpis will create further opportunity for G. austeni and, consequently, trypanosomiasis needs to be considered. The experimental results of ESTERHUIZEN ET AL. [7] can be interpreted to lend credence to exactly such a theory. They could suggest intense competition between the two species, to the extent that G. brevipalpis may actually benefit from odourbaited targets should their density be sufficient to eliminate G. austeni only. The existance of a reciprocal effect on $G$. austeni may be worth investigating . It could be exploitable. Then again, what is observed could simply be a delayed invasion response or even random noise. That ESTERHUIZEN ET AL. [7] were simply not able to measure the target-related mortality of G. brevipalpis, owing to high diffusion rates, is an alternative conclusion.

The K. P. P. equation can be solved by way of the application of the finite element method for the spatial discretisation and a backward difference for the temporal discretisation. This same strategy augmented by the linearisation and iteration of the nonlinear term also worked well for Fisher's equation, with good convergence for the range of conditions investigated. There is a certain amount of academic interest in this more challenging mathematics in that, if the numerical techniques employed are powerful enough to solve a nonlinear Fisher's equation, they will, logically, solve an equation with any other variants of the logistic term contemplated. This has important implications for the modelling of other vector-bourne diseases.

A comparison of the results of the two models offered some interesting insights. One concern at the outset was that if the age profile is altered in such a way that it contains a significantly higher proportion of pre-ovulatory flies, then the logistic growth rate (which is based on a fixed age profile) may no longer be appropriate. Just how reasonable is the assumption of a fixed age profile? Fisher's equation makes a far worse assumption in that it not only denies the existance of the pre-ovulatory stage, it also denies the existance of the entire pupal phase. A comparison between results of the two models might indicate the extent of the problem. The combination of warm temperatures, low imposed mortalities and long two year cycles gave

\footnotetext{
${ }^{2}$ Although this could be an artefact of assuming more mobile species have the same growth rate as more sedentary species.
} 
the population ample time to re-equilibrate in this particular case study, with the result that there was no discernable difference between the K.P.P.-model results and those arising from the unquestioning application of Fisher's equation (although this was not necessarily the case at lower temperatures). The suggestion is, therefore, that the assumption of a fixed age profile (in light of the longer than usual first interlarval period and artificially imposed mortalities) is permittable. If circumstances permit the denial of the existance of the pupal phase (Fisher's equation), then it stands to reason that a failure to recognize the existance of the far shorter pre-ovulatory phase (K.P.P. and Fisher's equation) should be permitted. Finally, convergence with little, or no iteration for Fisher's equation was useful in suggesting the steady state.

It is, nonetheless, inadviseable to use a model based on an unmodified Fisher's equation for tsetse. Lower temperatures or catastrophic mortalities inflicted, for example, by an aerial spray, are all circumstances in which attributing subsequent growth to a current, as opposed to historical, population would be profoundly incorrect. Notice that the model based on historical parentage would, under the latter circumstance, still fail to take the subsequent reproductive phase entrainment and altered age profile into account. Reproductive rates would initially be over-estimated, later, under-estimated and so on. Unlike Fisher's equation, however, the model is expected to recover. So long as circumstances allow the population to re-equilibrate there are unlikely to be any problems.

\section{Acknowledgements}

Abdalla Latif and the Onderstepoort Veterinary Institute are thanked for their generousity in both facilitating and funding this research. Guy Hendrickx is gratefully acknowledged for donating the two maps of tsetse risk and Andrew Parker is thanked for the information on puparial durations. Other, general information on the Hluhluwe-iMfolozi game reserve was supplied by Ezemvelo K.Z.N. Wildlife and the satellite image was kindly supplied by Marina Faber of Peace Parks Foundation. Brian Williams is thanked for taking a general interest in this work and John Hargrove, for sharing his vast knowledge of tsetse. This work obviously relies on the monumental research efforts and pioneering work carried out by the cited authors.

\section{References}

[1] Anonymous. Notes for field studies of tsetse flies in East Africa. Technical report, East Africa High Comission, Nairobi, 1955.

[2] E. Bursell. Characteristics of spontaneous activity in tsetse flies. Nature, 228:286-287, 1970.

[3] S. J. Childs. The energetic implications of the time discretisation in implementations of the A.L.E. equations. International Journal of Numerical Methods in Fluids, 32(8):9791019, 2000. 
[4] S. J. Childs. A model of pupal water loss in Glossina. Mathematical Biosciences, 221:7790, 2009.

[5] S. J. Childs and B. D. Reddy. Finite element simulation of the motion of a rigid body in a fluid with free surface. Computer Methods in Applied Mechanics and Engineering, 175(1-2):99-120, 1999.

[6] J. Esterhuizen. By communication. 2009.

[7] J. Esterhuizen, K. Kappmeier Green, E. M. Nevill, and P. Van Den Bossche. Selective use of odour-baited, insecticide-treated targets to control tsetse flies Glossina austeni and $G$. brevipalpis in South Africa. Medical and Veterinary Entomology, 20:464-469, 2006.

[8] Ezemvelo K.Z.N. Wildlife. Various online pamphlets and brochures. 2009.

[9] J. Ford and K. M. Katondo. The distribution of tsetse flies in Africa (3 maps). OAU, Cook, Hammond \& Kell, Nairobi, 1977.

[10] J. P. Glasgow. The Distribution and Abundance of Tsetse. International Series of Monographs on Pure and Applied Biology. Pergamon Press, 1963.

[11] J. W. Hargrove. Optimized simulation of the control of tsetse flies, Glossina pallidipes and G. m. morsitans (Diptera: Glossinidae) using odour-baited targets in Zimbabwe. Bulletin of Entomological Research, 93:19-29, 2003.

[12] J. W. Hargrove. The Trypanosomiases. Editors: I. Maudlin, P. H. Holmes and P. H. Miles. CABI publishing, Oxford, U.K., 2004.

[13] J. W. Hargrove. By communication. 2009.

[14] J. W. Hargrove, M. T. P. Holloway, G. A. Vale, A. J. E. Gough, and D. J. Hall. Catches of tsetse flies (Glossina spp.) (Diptera: Glossinidae) from traps baited with large doses of natural and synthetic host odour. Bulletin of Entomological Research, 85:215-227, 1995.

[15] J. W. Hargrove, S. J. Torr, and H. M. Kindness. Insecticide-treated cattle against tsetse (Diptera: Glossinidae): What governs success? Bulletin of Entomological Research, 93:203-217, 2003.

[16] Guy Hendrickx. Tsetse in Kwazulu Natal - an update -. Technical report, Agricultural and Veterinary Intelligence Analysis, 2007.

[17] C. H. N. Jackson. An artificially isolated generation of tsetse-flies (Diptera). Bulletin of Entomological Research, 37:291-299, 1946.

[18] J. E. Marsden and T. J. R. Hughes. Mathematical Foundations of Elasticity. Prentice-Hall, 1983.

[19] M. Y. Motloang, J. Masumu, P. Van Den Bossche, P. A. O. Majiwa, and A. A. Latif. Vector competance of field and colony Glossina austeni and Glossina brevipalpis for trypanosome species in KwaZulu-Natal. Journal of the South African Veterinary Association, 80(2):126-140, 2009. 
[20] J. D. Murray. Mathematical Biology. Springer Verlag, Berlin and Heidelberg, 1989.

[21] A. Parker. By communication. 2008.

[22] R. J. Phelps and P. M. Burrows. Puparial duration in Glossina morsitans orientalis under conditions of constant temperature. Entomologia Experimentalis et Applicata, 12:33-43, 1969.

[23] D. J. Rogers and T. P. Robinson. The Trypanosomiases. Editors: I. Maudlin, P. H. Holmes and P. H. Miles. CABI publishing, Oxford, U.K., 2004.

[24] David Rogers. Study of a natural population of Glossina fuscipes fuscipes Newstead and a model of fly movement. Journal of Animal Ecology, 46:309-330, 1977.

[25] David J. Rogers and Sarah E. Randolph. Estimation of rates of predation on tsetse. Medical and Veterinary Entomology, 4:195-204, 1990.

[26] R. E. Schulze and M. Maharaj. South African Atlas of Climatology and Agrohydrology. Water Research Commision, Pretoria, RSA, WRC report 1489/1/06, 2007.

[27] G. Vale. By communication. 2008.

[28] G. A. Vale, J. W. Hargrove, G. F. Cockbill, and R. J. Phelps. Field trials of baits to control populations of Glossina morsitans morsitans Westwood and G. pallidipes Austen (Diptera: Glossinidae). Bulletin of Entomological Research, 76:179-193, 1986.

[29] Brian Williams, Robert Dransfield, and Robert Brightwell. The control of tsetse flies in relation to fly movement and trapping efficiency. Journal of Applied Ecology, 29:163179, 1992.

[30] Brian G. Williams, Robert D. Dransfield, and Robert Brightwell. Tsetse fly (Diptera: Glossinidae) population dynamics and the estimation of mortality rates from life-table data. Bulletin of Entomological Research, 80:479-485, 1990.

\section{Addendum}

\section{The Change in Population Density Due to Migration}

Consider the hypothetical scenario of a mobile population in the absence of either reproduction or mortality (external and artificially imposed, or otherwise). Let $\Omega(t)$ be an arbitrary sub- 
volume of flies with boundary $\Gamma(t)$. Then biomass should be conserved so that

$$
\begin{aligned}
& \frac{D}{D t} \int_{\Omega(t)} \rho d \Omega=0 \quad \text { (rate of change of mass with time }=0 \text { ) } \\
& \frac{d}{d t} \int_{\Omega_{0}} \rho_{0} J_{0} d \Omega_{0}=0 \quad \text { (reformulating in the material } \\
& \text { configuration, } \left.\Omega_{0}\right) \\
& \int_{\Omega_{0}} \frac{d}{d t}\left\{\rho_{0} J_{0}\right\} d \Omega_{0}=0 \quad \text { (since limits are not time dependent } \\
& \text { in the material configuration) } \\
& \int_{\Omega_{0}}\left(\rho_{0} \dot{J}_{0}+\dot{\rho}_{0} J_{0}\right) d \Omega_{0}=0 \quad \text { (by the product rule) } \\
& \int_{\Omega(t)}(\dot{\rho}+\rho \operatorname{div} \mathbf{v}) d \Omega=0 \quad\left(\text { using } \dot{J}_{0}=J_{0} \operatorname{div} \mathbf{v}\right)^{\dagger},
\end{aligned}
$$

in which $\mathbf{v}$ is velocity. Since the volume was arbitrary it follows that the integrand must be zero. That is

$$
\dot{\rho}+\rho \operatorname{div} \mathbf{v}=0
$$

Now consider this biomass conservation in the context of another arbitrary sub-volume, this time of habitat, $\Omega_{h}$, with boundary $\Gamma_{h}$.

$$
\begin{array}{rlrl}
\int_{\Omega_{h}}\left(\frac{\partial \rho}{\partial t}+\nabla \rho \cdot \mathbf{v}+\rho \operatorname{div} \mathbf{v}\right) d \Omega_{h} & =0 & \text { (expanding } \dot{\rho} \text { ) } \\
\int_{\Omega_{h}}\left(\frac{\partial \rho}{\partial t}+\operatorname{div}\{\rho \mathbf{v}\}\right) d \Omega_{h} & =0 & \text { (by the product rule) } \\
\int_{\Omega_{h}} \frac{\partial \rho}{\partial t} d \Omega_{h}+\int_{\Gamma_{h}} \rho \mathbf{v} \cdot \mathbf{n} d \Gamma_{h} & =0 & \text { (by the divergence theorem) } \\
\int_{\Omega_{h}} \frac{\partial \rho}{\partial t} d \Omega_{h}-\int_{\Gamma_{h}}-\lambda \nabla \rho \cdot(-\mathbf{n}) d \Gamma_{h} & =0 & & \text { (by Fick's 1st law) } \\
\int_{\Omega_{h}}\left(\frac{\partial \rho}{\partial t}-\lambda \operatorname{div} \nabla \rho\right) d \Omega_{h} & =0 & \text { (by the divergence theorem). }
\end{array}
$$

Since the volume was arbitrary it again follows that the integrand must be zero. That is

$$
\frac{\partial \rho}{\partial t}=\lambda \operatorname{div} \nabla \rho
$$

in which $\lambda$ is the diffusion coefficient.

\footnotetext{
${ }^{\dagger}$ The material derivative of the Jacobian is given by the kinematic relation $\dot{J}_{0}=J_{0}$ div $\mathbf{v}$, a result demonstrated in most popular textbooks on continuum mechanics (eg. MARSDEN and HUGHES [18]).
} 


\section{Dimensionless Form}

Equation 1 is converted to its dimensionless form, as is standard practice before commencing a computation of this nature. (One wouldn't want the solution to be influenced in any way by the choice of units.) Suppose that $T$ is (only for the present) an unit of time, $X$ is a unit of length and $\eta$ is an unit of population density. The scaled variables are then

$$
\mathbf{x}=\overline{\mathbf{x}} X, \quad t=\bar{t} T \quad \text { and } \quad \rho=\bar{\rho} \eta \quad \text { (including } K=\bar{K} \eta \text { ). }
$$

Thus Equation 1 can be rewritten as

$$
\frac{\eta}{T} \frac{\partial \bar{\rho}}{\partial \bar{t}}=\frac{\eta}{X^{2}} \lambda \operatorname{div} \bar{\nabla} \bar{\rho}+r \eta \bar{\rho}^{*}\left(1-\frac{\bar{\rho}^{*}}{\bar{K}}\right)-\delta \eta \bar{\rho}
$$

All of this suggests using $T=\frac{X^{2}}{\lambda}$ and $X=\sqrt{\frac{\lambda}{r}}$ so that the above equation becomes

$$
\frac{\partial \bar{\rho}}{\partial \bar{t}}=\operatorname{div} \bar{\nabla} \bar{\rho}+\bar{\rho}^{*}\left(1-\frac{\bar{\rho}^{*}}{\bar{K}}\right)-\frac{\delta}{r} \bar{\rho}
$$

If the mesh is in units of kilometres, for example, then it must be converted by dividing through by $\sqrt{\frac{\lambda}{r}}$ kilometres.

Complication: If one intends accomodating any environmental variation in the rates of diffusion and growth, the scaled equation will entail different and therefore irreconcileable time steps. The equation

$$
\frac{\partial \bar{\rho}}{\partial \bar{t}}=\frac{\lambda}{\lambda_{\text {scale }}} \operatorname{div} \bar{\nabla} \bar{\rho}+\frac{r}{r_{\text {scale }}} \bar{\rho}^{*}\left(1-\frac{\bar{\rho}^{*}}{\bar{K}}\right)-\frac{\delta}{r_{\text {scale }}} \bar{\rho}
$$

allows a time discretisation which conforms.

\section{Variational Formulation}

A variational formulation of Equation 4 is obtained in the usual fashion; premultiplying the primitive variable equation by an arbitrary function, $w$, and integrating over the domain, $\Omega$, gives rise to the equation

$$
\int_{\Omega} w \frac{\partial \rho}{\partial t} d \Omega=\frac{\lambda}{\lambda_{\text {scale }}} \int_{\Omega} w \operatorname{div} \nabla \rho d \Omega+\frac{r}{r_{\text {scale }}} \int_{\Omega} w \rho^{*}\left(1-\frac{\rho^{*}}{K}\right) d \Omega-\frac{\delta}{r_{\text {scale }}} \int_{\Omega} w \rho d \Omega .
$$

The approximation-wise cumbersome second derivatives can also be avoided in the usual fashion. The term which contains the divergence of $\nabla \rho$ can be regarded as one part of a differentiated product and the divergence theorem applied so that

$$
\int_{\Omega} w \frac{\partial \rho_{, i}}{\partial x_{i}} d \Omega=\int_{\Gamma} w \rho_{, i} n_{i} d \Gamma-\int_{\Omega} w_{, i} \rho_{, i} d \Omega,
$$


in which $\mathbf{n}$ is the outward unit normal and $\Gamma$ is the domain boundary. The boundary integral obviously vanishes for a von Neumann, $\mathbf{n} \cdot \nabla \rho=0$ type boundary condition while the arbitrary vector of the formulation can be assigned a value of zero where boundary conditions are Dirichlet (and an equation is consequently not required). The boundary integral is therefore irrelevant. 